\title{
Acoustic field of a pulsating cylinder in a rarefied gas: Thermoviscous and curvature effects
}

\author{
Y. Ben Ami and A. Manela* \\ Faculty of Aerospace Engineering, Technion-Israel Institute of Technology, Haifa 32000, Israel \\ (Received 10 July 2017; published 21 September 2017)
}

\begin{abstract}
We study the acoustic field of a circular cylinder immersed in a rarefied gas and subject to harmonic small-amplitude normal-to-wall displacement and heat-flux excitations. The problem is analyzed in the entire range of gas rarefaction rates and excitation frequencies, considering both single cylinder and coaxial cylinders setups. Numerical calculations are carried out via the direct simulation Monte Carlo method, applying a noniterative algorithm to impose the boundary heat-flux condition. Analytical predictions are obtained in the limits of ballistic- and continuum-flow conditions. Comparing with a reference inviscid continuum solution, the results illustrate the specific impacts of gas rarefaction and boundary curvature on the acoustic source efficiency. Inspecting the far-field properties of the generated disturbance, the continuum-limit solution exhibits an exponential decay of the signal with the distance from the source, reflecting thermoviscous effects, and accompanied by an inverse square-root decay, characteristic of the inviscid problem. Stronger attenuation is observed in the ballistic limit, where boundary curvature results in "geometric reduction" of the molecular layer affected by the source, and the signal vanishes at a distance of few acoustic wavelengths from the cylinder. The combined effects of mechanical and thermal excitations are studied to seek for optimal conditions to monitor the vibroacoustic signal. The impact of boundary curvature becomes significant in the ballistic-flow regime, where the optimal heat-flux amplitude required for sound reduction decreases with the distance from the source and is essentially a function of the acoustic-wavelength-scaled distance only.
\end{abstract}

DOI: 10.1103/PhysRevFluids.2.093401

\section{INTRODUCTION}

The pressure field generated by small-amplitude motions of an object in a quiescent fluid is a classical problem in acoustics, which has been considered extensively over the years in various model configurations [1]. Traditionally, continuum conditions are assumed, and the far-field source radiation is described in terms of its overall strength and directivity field. The calculation is regularly carried out for an ideal compressible fluid, where the attenuating effects of viscosity and thermal dissipation are neglected. In an effort to complement these works, more recent studies (e.g., Refs. [2,3]) have examined the impacts of fluid viscosity and body shear stress on sound generation. Yet, homentropic flow conditions have been assumed at the outset, resulting in decoupling of the dynamic and thermodynamic flow descriptions.

In parallel with continuum studies, the propagation of sound waves in rarefied gases has been investigated in a considerable number of works. These studies become relevant wherever the characteristic lengthscale, or timescale, of the problem involved is of the order of molecular mean free path, or time, respectively. Starting in the middle of the 20th Century, and continuing to current research, almost all investigations have focused on sound generation by a planar vibrating surface (e.g., Refs. [4-9] and papers cited therein). Considering a similar planar setup, other studies have examined the pressure perturbation generated by thermoacoustic surface excitation, in the form of time temperature variations of the boundary [10-13]. A later work has then indicated that it may

*amanela@technion.ac.il 
be more practical to monitor the heat flux at a boundary rather than its temperature [14]. Applying such a condition, it has been demonstrated that the vibroacoustic signal of an oscillating plane may be monitored by counter-phase thermal excitation of the surface $[14,15]$.

While the planar setup is of primary significance, it is of evident interest to examine the propagation of acoustic waves in rarefied gases at qualitatively different two- and three-dimensional model configurations, as was done in continuum acoustics [1]. Apart from the fundamental interest in such studies, these investigations should shed light on the propagation of acoustic waves in a variety of practical micromechanical setups, where small length- and time-scales are encountered. Toward this end, Yap and Sader [16] have examined the acoustic field induced by a rigid sphere oscillating in a rarefied gas. Somewhat earlier, Kalempa and Sharipov [17] have investigated the thermoacoustic wave generation by the unsteady heating of a cylinder in a coaxial-cylinders setup. To the best of our knowledge, these are the existing examples (excluding studies on the near-field properties in a nonplanar configuration, e.g., Ref. [18]) where the acoustic field of a nonplanar object, immersed in a quiescent rarefied gas, has been theoretically considered.

In light of the above, the objective of the present work is to analyze the mechanism of sound generation in a canonical "pulsating cylinder" setup immersed in a rarefied gas. Both single and coaxial cylinders configurations are considered, and the acoustic disturbance is generated via either mechanical (normal boundary displacement) or thermal (normal heat-flux perturbation) excitations. Cylinders radii and excitation timescales are chosen so that noncontinuum flow conditions prevail, and the problem is considered in the entire range of gas rarefaction rates. By considering the cylindrical setup, we aim at analyzing the efficiency of a two-dimensional acoustic monopole at rarefied-flow conditions and seek to compare it with the known solution for an expanding cylinder in an ideal fluid [1]. Analytical investigations are carried out in the two limits of low (continuum) and high (free-molecular) rarefaction rates and are accompanied by direct simulation Monte Carlo (DSMC) calculations in the intermediate range of gas rarefaction.

The paper starts with a general statement of the problem, given in Sec. II. The analysis is described in Sec. III, where the analytical and numerical schemes of solution are presented. Our results are given in Sec. IV, for both single- and double-cylinder configurations, and the impact of combining the mechanical and thermal excitations on the total system radiation is discussed. Concluding comments are given in Sec. V.

\section{STATEMENT OF THE PROBLEM}

Consider a monoatomic gas layer of uniform density $\rho_{0}^{*}$ (with an asterisk marking a dimensional quantity) confined between two infinitely long coaxial cylinders. The inner and outer cylinders are of radii $r^{*}=r_{1}^{*}$ and $r^{*}=r_{2}^{*}$, respectively. The gas is initially at rest and in thermodynamic equilibrium with the diffuse reflecting boundaries, which, at time $t^{*}<0$, maintain a common temperature $T_{0}^{*}$. At time $t^{*} \geqslant 0$, the inner cylinder pulsates in the radial direction with a prescribed velocity profile,

$$
\varepsilon \mathbf{U}_{1}^{*}\left(t^{*}\right)=\varepsilon U_{1}^{*}\left(t^{*}\right) \hat{\mathbf{r}},
$$

while the outer cylinder is kept stationary $\left(\mathbf{U}_{2}^{*} \equiv \mathbf{0}\right)$. Simultaneously, the radial heat-flux at the inner wall is imposed,

$$
\varepsilon \mathbf{Q}_{1}^{*}\left(t^{*}\right)=\varepsilon Q_{1}^{*}\left(t^{*}\right) \hat{\mathbf{r}},
$$

and the outer cylinder is maintained adiabatic $\left(\mathbf{Q}_{2}^{*} \equiv \mathbf{0}\right)$. In Eqs. (1) and (2), it is assumed that $\varepsilon \ll 1$, so that the system description may be linearized about its initial equilibrium state.

Rather than imposing the boundary temperature, Eq. (2) prescribes the walls normal heat flux. This follows a set of recent works, where it has been noted that, in practice, the surface temperature at any experimental setup (particularly if varying with time) can only be monitored indirectly through the direct imposition of boundary heat-flux. In such a formulation, the boundary temperature is treated as unknown, and calculated in the process of analysis. Following this approach, Gospodinov, Roussinov, and Stefanov [19] have investigated the impact of changing between isothermal and 
adiabatic conditions on the oscillatory cylindrical Couette flow at high rotation speeds. Manela and Pogorelyuk $[14,15]$ have considered the effect of thermal boundary conditions on the passage of sound waves in a planar setup, suggesting it as a thermoacoustic means for active noise control of a vibrating surface. Meng, Zhang, and Reese [20] have studied numerically the effect of heat-flux boundary conditions on rarefied Couette and force-driven Poiseuille flows. And, most recently, boundary-heat-flux monitoring (in the form of "instantaneous insulation" of a microgap) has been suggested as a means for flow generation at the microscale [21]. In line with these studies, the present investigation applies a similar thermal condition on the cylindrical walls.

Our problem is rendered dimensionless by normalizing the position by the inner wall radius $r_{1}^{*}$, the velocity by the mean thermal speed of a gas molecule $U_{\text {th }}^{*}=\sqrt{2 \mathcal{R}^{*} T_{0}^{*}}$ (with $\mathcal{R}^{*}$ denoting the specific gas constant), and the time by the consequent time-scale $r_{1}^{*} / U_{\mathrm{th}}^{*}$. Density and temperature are scaled by $\rho_{0}^{*}$ and $T_{0}^{*}$, respectively, and the heat flux is normalized by $\rho_{0}^{*} U_{\mathrm{th}}^{* 3}$. The nondimensional problem is governed by the scaled excitation profiles $U_{1}(t)$ and $Q_{1}(t)$, and by the parameters

$$
R=r_{2}^{*} / r_{1}^{*} \text { and } \mathrm{Kn}=l^{*} / r_{1}^{*},
$$

marking the cylinders radii ratio and radius-based Knudsen number, respectively. In Eq. (3), $l^{*}$ denotes the mean free path of a gas molecule.

In what follows, we investigate the gas response to the mechanical and thermal excitations specified in Eqs. (1) and (2), in both double- and single-cylinder setups. The single-cylinder configuration follows from the above by removing the outer cylinder or by taking its radius to be large compared with the "penetration length" of the acoustic signal, to enable Monte Carlo calculations in a finite domain. The analysis is carried out over the entire range of gas rarefaction rates, combining analytic ballistic- and continuum-limit predictions with numerical Monte Carlo calculations.

\section{ANALYSIS}

\section{A. Numerical scheme: DSMC method}

The direct simulation Monte Carlo method, proposed by Bird [22], is a stochastic particle method commonly applied for analyzing rarefied gas flows. In the present work, the method is used to obtain results that are "numerically exact" at arbitrary rarefied-flow conditions, and to validate our analytical ballistic- and continuum-limit solutions. We adopt Bird's algorithm in one-dimensional cylindrical coordinates [22] and assume hard-sphere interactions between the gas molecules. The boundaries are taken fully diffuse, with prescribed radial velocity and heat-flux. In difference from traditional application of the wall-interaction algorithm, the walls temperatures are treated as unknown. Application of the heat-flux conditions therefore requires modification in the conventional calculation.

Imposition of a heat-flux condition in DSMC calculations has been recently discussed in several works, where iterative algorithms have been suggested to comply with the thermal constraint $[14,15,23,24]$. In difference from these works, we follow here an exact (noniterative) algorithm, based on an energy balance occurring at the boundary. A similar approach has been applied in Ref. [20] in the context of a finite difference discrete-velocity calculation. Requiring no iterative procedure, this scheme appears advantageous and more efficient for numerical implementation.

To describe the algorithm, consider the distribution of the (dimensional) normal and tangential velocity components for the wall-reflected molecules at the $i$ th time step,

$$
v_{\perp}^{*^{i}}=\frac{1}{\beta_{w}^{*^{i}}} \sqrt{-\ln \left(R_{U}\right)}+U_{w}^{*^{i}} \text { and } v_{\|}^{*^{i}}=\frac{1}{\sqrt{2} \beta_{w}^{*^{i}}} R_{G},
$$

respectively. In Eq. (4), $R_{U} \in[0,1]$ and $R_{G} \in(-\infty, \infty)$ are uniformly- and Gaussian-distributed random numbers, $U_{w}^{*^{i}}$ is the normal wall velocity, and $\beta_{w}^{*^{i}}=\sqrt{1 / 2 \mathcal{R}^{*} T_{w}^{*^{i}}}$. Assigning a heat-flux condition, the value of $\beta_{w}^{i}$ is a priori unknown at every time step, and should be determined. For the 
fully diffuse boundary considered here, the total mass and thermal energy fluxes of the molecules reflected at the boundary are

$$
\begin{aligned}
\mathcal{M}_{\mathrm{out}}^{*^{i}} & =\frac{\rho_{w}^{*^{i}}\left(\beta_{w}^{*^{i}}\right)^{3}}{\pi^{3 / 2}} \int_{\left(\xi_{r}^{*}-U_{w}^{*^{i}}\right)>0}\left(\xi_{r}^{*}-U_{w}^{*^{i}}\right) \exp \left\{-\left(\beta_{w}^{*^{i}}\right)^{2}\left[\left(\xi_{r}^{*}-U_{w}^{*^{i}}\right)^{2}+\xi_{\theta}^{*^{2}}+\xi_{z}^{*^{2}}\right]\right\} d \xi^{*} \\
& =\frac{\rho_{w}^{*^{i}}}{2 \beta_{w}^{*^{i}} \sqrt{\pi}}
\end{aligned}
$$

and

$$
\begin{aligned}
\mathcal{H}_{\mathrm{out}}^{*^{i}}= & \frac{\rho_{w}^{*^{i}}\left(\beta_{w}^{*^{i}}\right)^{3}}{2 \pi^{3 / 2}} \int_{\left(\xi_{r}^{*}-U_{w}^{*^{i}}\right)>0}\left(\xi_{r}^{*}-U_{w}^{*^{i}}\right)\left[\left(\xi_{r}^{*}-U_{w}^{* i}\right)^{2}+\xi_{\theta}^{*^{2}}+\xi_{z}^{*^{2}}\right] \\
& \times \exp \left\{-\left(\beta_{w}^{*^{i}}\right)^{2}\left[\left(\xi_{r}^{*}-U_{w}^{*^{i}}\right)^{2}+\xi_{\theta}^{*^{2}}+\xi_{z}^{*^{2}}\right]\right\} d \xi^{*}=\frac{\rho_{w}^{*^{i}}}{2\left(\beta_{w}^{*^{i}}\right)^{3} \sqrt{\pi}},
\end{aligned}
$$

respectively. Combining Eqs. (5) and (6), we find

$$
\beta_{w}^{*^{i}}=\sqrt{\frac{\mathcal{M}_{\mathrm{out}}^{*^{i}}}{\mathcal{H}_{\mathrm{out}}^{*^{i}}}}
$$

Applying the conservation of mass, $\mathcal{M}_{\text {out }}^{*^{i}}=\mathcal{M}_{\mathrm{in}}^{*^{i}}$, and thermal energy, $\mathcal{H}_{\mathrm{out}}^{*^{i}}=\mathcal{H}_{\mathrm{in}}^{*^{i}}+Q_{w}^{*^{i}}$, at the boundary, yield

$$
\beta_{w}^{*^{i}}=\sqrt{\frac{\mathcal{M}_{i n}^{*^{i}}}{\mathcal{H}_{\mathrm{in}}^{* i}+Q_{w}^{*^{i}}}}
$$

where $\mathcal{M}_{\text {in }}^{*^{i}}$ and $\mathcal{H}_{\text {in }}^{*^{i}}$ are computed at each time step via

$$
\mathcal{M}_{\mathrm{in}}^{*^{i}}=\frac{N}{2 \pi r_{w}^{*} \Delta t^{*}} \text { and } \mathcal{H}_{\mathrm{in}}^{*^{i}}=\frac{1}{2 \pi r_{w}^{*} \Delta t^{*}} \sum_{j=1}^{N}\left[\left(\xi_{r}^{*^{i, j}}-U_{w}^{*^{i}}\right)^{2}+\left(\xi_{\theta}^{*^{i, j}}\right)^{2}+\left(\xi_{z}^{*^{i, j}}\right)^{2}\right] .
$$

In Eq. (9), summation is carried over all $j=1, \ldots, N$ particles that have collided with the boundary during the $\Delta t^{*}$ time interval, and $r_{w}^{*}$ is the cylindrical wall radius. Having determined $\mathcal{M}_{\mathrm{in}}^{*^{i}}$ and $\mathcal{H}_{\mathrm{in}}^{*^{i}}$, $\beta_{w}^{*^{i}}$ is obtained using Eq. (8). Each of the reflected particles is then assigned a velocity according to Eq. (4), and the simulation is followed to the next time step. Notably, Eq. (8) remains valid for arbitrary nonsmall heat-fluxes, and the algorithm may be easily extended to simulate two- and three-dimensional setups.

An outline of our computational procedure now follows. In each calculation, the one-dimensional computational grid has been divided into 50-350 cells of length $\Delta r^{*}$ (sufficient to capture the acoustic signal), and a time step of $\Delta t^{*}=0.2 \Delta r^{*} / U_{\mathrm{th}}^{*}$ has been assigned. For the harmonic wall excitation studied hereafter, the computation has been carried out until the system has achieved its final periodic state. To simulate a single-cylinder setup (see Sec. IV A), the outer adiabatic cylinder has been placed far enough, so that it had no effect on the system response. A single run consisted of $2 \times 10^{7}-5 \times 10^{7}$ particles, and 50 realizations have been carried out to sufficiently reduce the numerical noise in the calculated hydrodynamic fields. Each simulation lasted 1-24 h (depending on the system Knudsen number) using an eight-cores Intel i7-6700 machine.

\section{B. Ballistic-flow limit}

In the framework of the kinetic theory of gases and the axially symmetric configuration considered, the gas state is governed by the probability density function $f=f(r, t, \boldsymbol{\xi})$ of finding a gas molecule 
with velocity about $\xi$ at radial distance near $r$ at time $t$. At the linearized conditions assumed, we write

$$
f(r, t, \xi)=F[1+\varepsilon \phi(r, t, \xi)],
$$

where $F=\pi^{-3 / 2} \exp \left[-\xi^{2}\right]$ denotes the nondimensional Maxwellian equilibrium distribution, and $\phi(r, t, \xi)$ marks the probability perturbation function. Assuming the Knudsen number to be large, we analyze the collisionless Boltzmann equation for $\phi(r, t, \xi)$ in cylindrical coordinates [25],

$$
\frac{\partial \phi}{\partial t}+\xi_{r} \frac{\partial \phi}{\partial r}+\frac{\xi_{\theta}^{2}}{r} \frac{\partial \phi}{\partial \xi_{r}}-\frac{\xi_{r} \xi_{\theta}}{r} \frac{\partial \phi}{\partial \xi_{\theta}}=0 .
$$

Denoting $\xi_{2 \mathrm{D}}=\left(\xi_{r}^{2}+\xi_{\theta}^{2}\right)^{1 / 2}$ and $\theta_{\xi}=\tan ^{-1}\left(\xi_{\theta} / \xi_{r}\right)$, and changing variables between $\left(\xi_{r}, \xi_{\theta}\right)$ and $\left(\xi_{2 \mathrm{D}}, \theta_{\xi}\right)$, Eq. (11) is rewritten in the form

$$
\frac{\partial \phi}{\partial t}+\xi_{2 \mathrm{D}} \cos \theta_{\xi} \frac{\partial \phi}{\partial r}-\frac{\xi_{2 \mathrm{D}} \sin \theta_{\xi}}{r} \frac{\partial \phi}{\partial \theta_{\xi}}=0 .
$$

The equation is supplemented by the initial condition,

$$
\phi\left(r, t=0^{-}, \xi_{2 \mathrm{D}}, \theta_{\xi}, \xi_{z}\right)=0,
$$

together with a linearized form of the diffuse boundary conditions imposed at the cylindrical walls,

$$
\begin{gathered}
\phi\left(r=1,\left|\theta_{\xi}\right|<\pi / 2\right)=\rho_{1}(t)+2 \xi_{2 \mathrm{D}} \cos \theta_{\xi} U_{1}(t)+T_{1}(t)\left(\xi_{2 \mathrm{D}}^{2}+\xi_{z}^{2}-3 / 2\right) \\
\quad \text { and } \phi\left(r=R, t, \pi / 2<\left|\theta_{\xi}\right|<\pi\right)=\rho_{2}(t)+T_{2}(t)\left(\xi_{2 \mathrm{D}}^{2}+\xi_{z}^{2}-3 / 2\right) .
\end{gathered}
$$

In Eqs. (14), $\rho_{1,2}(t)$ and $T_{1,2}(t)$ are yet unknown, where $T_{1,2}(t)$ mark the time perturbations of walls temperatures. The conditions appearing in Eqs. (14) are of "half-space" type, thus applied to the reflected molecules at $r=1$ having $\left|\theta_{\xi}\right|<\pi / 2$ (i.e., $\xi_{r}-U_{1}(t)>0$ ), and at $r=R$ having $\pi / 2<\left|\theta_{\xi}\right|<\pi$ (i.e., $\xi_{r}<0$ ).

Making use of the time Laplace transform,

$$
\bar{g}(s)=\int_{0}^{\infty} g(t) \exp [-s t] d t,
$$

Eq. (12), in conjunction with the initial condition in Eq. (13), is transformed into the equation

$$
\xi_{2 \mathrm{D}} \cos \theta_{\xi} \frac{\partial \bar{\phi}}{\partial r}-\frac{\xi_{2 \mathrm{D}} \sin \theta_{\xi}}{r} \frac{\partial \bar{\phi}}{\partial \theta_{\xi}}=-s \bar{\phi} .
$$

Applying the method of characteristics, the solution for Eq. (16) is obtained by finding an integral surface $\mathcal{S}\left(r, \theta_{\xi}, \bar{\phi}\right)$ that is tangent at each point to the vector $\left(\xi_{2 \mathrm{D}} \cos \theta_{\xi},-\xi_{2 \mathrm{D}} \sin \theta_{\xi} / r,-s \bar{\phi}\right)$. This yields a pair of ordinary equations,

$$
\frac{d r}{d \theta_{\xi}}=-\frac{r}{\tan \theta_{\xi}}, \quad \frac{d \bar{\phi}}{d r}=-\frac{s}{\xi_{2 \mathrm{D}}} \frac{\bar{\phi}}{\cos \theta_{\xi}},
$$

where the first is amenable to the solution

$$
r=\frac{c_{1}}{\sin \theta_{\xi}}
$$

Substituting Eq. (18) into the second equation in Eq. (17) and integrating, yield

$$
\bar{\phi}=c_{2} \exp \left[\mp \frac{s}{\xi_{2 \mathrm{D}}} r \sqrt{1-\left(\frac{c_{1}}{r}\right)^{2}}\right],
$$

where the negative and positive signs refer to the $\theta_{\xi}$-intervals $\left|\theta_{\xi}\right| \leqslant \pi / 2$ and $\pi / 2<\left|\theta_{\xi}\right| \leqslant \pi$, respectively. The expression for $\bar{\phi}$ is specified by taking $c_{2}=g\left(c_{1}\right)=g\left(r \sin \theta_{\xi}\right)$ in Eq. (19), and 


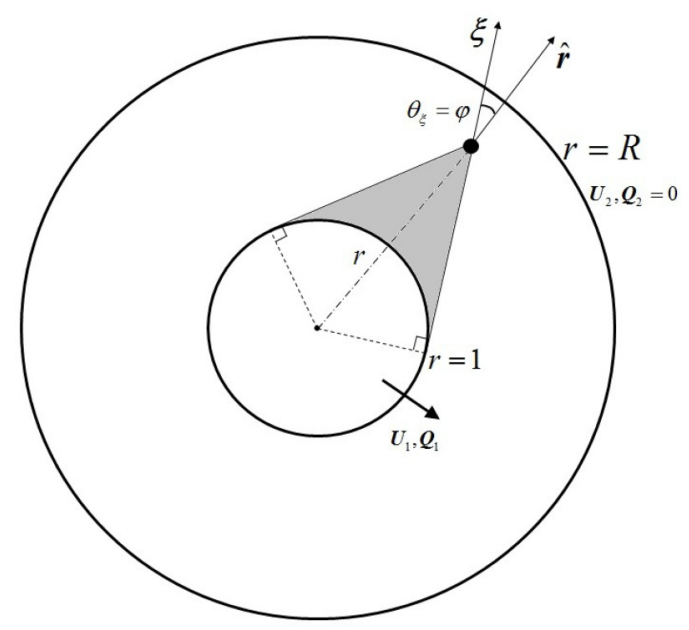

FIG. 1. Illustration of particle's $\theta_{\xi}$-parametrization in the ballistic limit for a double-cylinder setup. The bold point marks the position of an arbitrary particle, and the shaded and unshaded $\theta_{\xi}$-zones confine the $\theta_{\xi}$-sections acquired by the particle when reflected from the $r=1$ or $r=R$ boundaries, respectively.

applying the transformed form of boundary conditions in Eq. (14). This results in the double-sided solution

$$
\bar{\phi}=\left\{\begin{array}{l}
{\left[\bar{\rho}_{1}(s) \pm 2 \xi_{2 \mathrm{D}} \bar{U}_{1}(s) \sqrt{1-r^{2} \sin ^{2} \theta_{\xi}}+\bar{T}_{1}(s)\left(\xi_{2 \mathrm{D}}^{2}+\xi_{z}^{2}-\frac{3}{2}\right)\right]} \\
\quad \times \exp \left[-\frac{s}{\xi_{2 \mathrm{D}}}\left(r \cos \theta_{\xi} \mp \sqrt{1-r^{2} \sin ^{2} \theta_{\xi}}\right)\right] \\
{\left[\bar{\rho}_{2}(s)+\bar{T}_{2}(s)\left(\xi_{2 \mathrm{D}}^{2}+\xi_{z}^{2}-\frac{3}{2}\right)\right] \exp \left[-\frac{s}{\xi_{2 \mathrm{D}}}\left(r \cos \theta_{\xi} \mp \sqrt{R^{2}-r^{2} \sin ^{2} \theta_{\xi}}\right)\right],}
\end{array}\right.
$$

corresponding to particles having $\xi_{r}>0$ and $\xi_{r}<0$, respectively. Imposed by geometrical considerations, gas particles reflected from the convex $r=1$ cylinder acquire $\left|\theta_{\xi}\right| \leqslant \varphi$, where $\varphi=\sin ^{-1}(1 / r)$. Inversely, particles reflected from the concave $r=R$ surface are confined to $\varphi \leqslant\left|\theta_{\xi}\right| \leqslant \pi$. This is illustrated in Fig. 1, mapping the $\theta_{\xi}$-sections acquired by the indicated particle when reflected from the convex or concave walls.

Taking the inverse Laplace transform of Eq. (20), we find

$$
\phi\left(r, t, \xi_{2 \mathrm{D}}, \theta_{\xi}, \xi_{z}\right)=\left\{\begin{array}{l}
\rho_{1}\left(t_{r_{1}}\right)+2 \xi_{2 \mathrm{D}} U_{1}\left(t_{r_{1}}\right) \sqrt{1-r^{2} \sin ^{2} \theta_{\xi}}+T_{1}\left(t_{r_{1}}\right)\left(\xi_{2 \mathrm{D}}^{2}+\xi_{z}^{2}-\frac{3}{2}\right),\left|\theta_{\xi}\right| \leqslant \varphi \\
\rho_{2}\left(t_{r_{2}}\right)+T_{2}\left(t_{r_{2}}\right)\left(\xi_{2 \mathrm{D}}^{2}+\xi_{z}^{2}-\frac{3}{2}\right), \varphi \leqslant\left|\theta_{\xi}\right| \leqslant \pi
\end{array}\right.
$$

where

$$
t_{r_{1}}=t-(r-1) / \xi_{2 \mathrm{D}}=t-\left(r \cos \theta_{\xi}-\sqrt{1-r^{2} \sin ^{2} \theta_{\xi}}\right) / \xi_{2 \mathrm{D}}
$$

and

$$
t_{r_{2}}=t-(R-r) / \xi_{2 \mathrm{D}}=t-\left(r \cos \theta_{\xi}+\sqrt{R^{2}-r^{2} \sin ^{2} \theta_{\xi}}\right) / \xi_{2 \mathrm{D}}
$$

are the acoustic retarded times corresponding to the signals generated at $r=1$ and $r=R$, respectively. The positive and negative signs that should have preceded the square-root expressions in $t_{r_{1}}$ and $t_{r_{2}}$, respectively, have been omitted, as they represent molecules entering or leaving the domain through the inner and outer cylinders.

To determine the fields $\rho_{1,2}(t)$ and $T_{1,2}(t)$, the macroscopic conditions of no-penetration,

$$
\frac{1}{\pi^{3 / 2}} \int_{-\infty}^{\infty} d \xi_{z} \int_{0}^{\infty} d \xi_{2 \mathrm{D}} \int_{-\pi}^{\pi} \phi(r=1, R) \xi_{2 \mathrm{D}}^{2} \cos \theta_{\xi} \exp \left[-\xi_{2 \mathrm{D}}^{2}-\xi_{z}^{2}\right] d \theta_{\xi}=U_{1,2}(t),
$$


and heat-flux,

$$
\begin{aligned}
& \frac{1}{2 \pi^{3 / 2}} \int_{-\infty}^{\infty} d \xi_{z} \int_{0}^{\infty} d \xi_{2 \mathrm{D}} \int_{-\pi}^{\pi} \phi(r=1, R) \xi_{2 \mathrm{D}}^{2} \cos \theta_{\xi}\left(\xi_{2 \mathrm{D}}^{2}+\xi_{z}^{2}\right) \exp \left[-\xi_{2 \mathrm{D}}^{2}-\xi_{z}^{2}\right] d \theta_{\xi}-\frac{5}{4} U_{1,2}(t) \\
& \quad=Q_{1,2}(t)
\end{aligned}
$$

are imposed at the walls, where $U_{2}(t)=0$ and $Q_{2}(t)=0$. Substituting Eq. (21) into Eqs. (22) and (23), and carrying the changes of variables

$$
\tau=t-g_{1,2}\left(r, \theta_{\xi}\right) / \xi_{2 \mathrm{D}}
$$

with

$$
g_{1}\left(r, \theta_{\xi}\right)=r \cos \theta_{\xi}-\sqrt{1-r^{2} \sin ^{2} \theta_{\xi}} \text { and } g_{2}\left(r, \theta_{\xi}\right)=r \cos \theta_{\xi}+\sqrt{R^{2}-r^{2} \sin ^{2} \theta_{\xi}},
$$

yields the set of coupled integral equations,

$$
\begin{aligned}
& \rho_{1}(t)=\frac{7 \sqrt{\pi}}{8} U_{1}(t)-\sqrt{\pi} Q_{1}(t) \\
& +\frac{1}{\sqrt{\pi}} \int_{0}^{t} d \tau \int_{\pi / 2<\left|\theta_{\xi}\right|<\pi} \rho_{2}(\tau) \cos \theta_{\xi} \frac{\left(g_{2}\left(1, \theta_{\xi}\right)\right)^{3}}{(t-\tau)^{4}}\left[\frac{\left(g_{2}\left(1, \theta_{\xi}\right)\right)^{2}}{2(t-\tau)^{2}}-\frac{11}{4}\right] \exp \left[-\frac{\left(g_{2}\left(1, \theta_{\xi}\right)\right)^{2}}{(t-\tau)^{2}}\right] d \theta_{\xi} \\
& +\frac{1}{\sqrt{\pi}} \int_{0}^{t} d \tau \int_{\pi / 2<\left|\theta_{\xi}\right|<\pi} T_{2}(\tau) \cos \theta_{\xi} \frac{\left(g_{2}\left(1, \theta_{\xi}\right)\right)^{3}}{(t-\tau)^{4}}\left[\frac{\left(g_{2}\left(1, \theta_{\xi}\right)\right)^{4}}{2(t-\tau)^{4}}-\frac{13\left(g_{2}\left(1, \theta_{\xi}\right)\right)^{2}}{4(t-\tau)^{2}}+3\right] \\
& \times \exp \left[-\frac{\left(g_{2}\left(1, \theta_{\xi}\right)\right)^{2}}{(t-\tau)^{2}}\right] d \theta_{\xi} \\
& T_{1}(t)=\frac{\sqrt{\pi}}{4} U_{1}(t)+2 \sqrt{\pi} Q_{1}(t) \\
& +\frac{1}{\sqrt{\pi}} \int_{0}^{t} d \tau \int_{\pi / 2<\left|\theta_{\xi}\right|<\pi} \rho_{2}(\tau) \cos \theta_{\xi} \frac{\left(g_{2}\left(1, \theta_{\xi}\right)\right)^{3}}{(t-\tau)^{4}}\left[-\frac{\left(g_{2}\left(1, \theta_{\xi}\right)\right)^{2}}{(t-\tau)^{2}}+\frac{3}{2}\right] \\
& \times \exp \left[-\frac{\left(g_{2}\left(1, \theta_{\xi}\right)\right)^{2}}{(t-\tau)^{2}}\right] d \theta_{\xi} \\
& +\frac{1}{\sqrt{\pi}} \int_{0}^{t} d \tau \int_{\pi / 2<\left|\theta_{\xi}\right|<\pi} T_{2}(\tau) \cos \theta_{\xi} \frac{\left(g_{2}\left(1, \theta_{\xi}\right)\right)^{3}}{(t-\tau)^{4}}\left[-\frac{\left(g_{2}\left(1, \theta_{\xi}\right)\right)^{4}}{(t-\tau)^{4}}+\frac{5\left(g_{2}\left(1, \theta_{\xi}\right)\right)^{2}}{2(t-\tau)^{2}}-2\right] \\
& \times \exp \left[-\frac{\left(g_{2}\left(1, \theta_{\xi}\right)\right)^{2}}{(t-\tau)^{2}}\right] d \theta_{\xi} \\
& \rho_{2}(t)=\frac{1}{\sqrt{\pi}} \int_{0}^{t} d \tau \int_{\left|\theta_{\xi}\right|<\pi / 2} \rho_{1,2}(\tau) \cos \theta_{\xi} \frac{\left(g_{1,2}\left(R, \theta_{\xi}\right)\right)^{3}}{(t-\tau)^{4}}\left[\frac{\left(g_{1,2}\left(R, \theta_{\xi}\right)\right)^{2}}{2(t-\tau)^{2}}-\frac{11}{4}\right] \\
& \times \exp \left[-\frac{\left(g_{1,2}\left(R, \theta_{\xi}\right)\right)^{2}}{(t-\tau)^{2}}\right] d \theta_{\xi}+\frac{1}{\sqrt{\pi}} \int_{0}^{t} d \tau \int_{\left|\theta_{\xi}\right|<\varphi_{m}} U_{1}(\tau) \cos \theta_{\xi} \frac{\left(g_{1}\left(R, \theta_{\xi}\right)\right)^{4}}{(t-\tau)^{5}}
\end{aligned}
$$

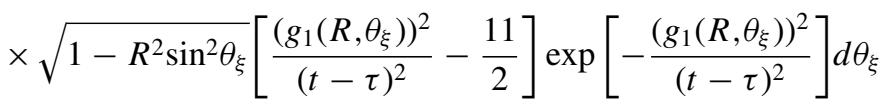

$$
\begin{aligned}
& +\frac{1}{\sqrt{\pi}} \int_{0}^{t} d \tau \int_{\left|\theta_{\xi}\right|<\pi / 2} T_{1,2}(\tau) \cos \theta_{\xi} \frac{\left(g_{1,2}\left(R, \theta_{\xi}\right)\right)^{3}}{(t-\tau)^{4}}\left[\frac{\left(g_{1,2}\left(R, \theta_{\xi}\right)\right)^{4}}{2(t-\tau)^{4}}-\frac{13\left(g_{1,2}\left(R, \theta_{\xi}\right)\right)^{2}}{4(t-\tau)^{2}}+3\right] \\
& \times \exp \left[-\frac{\left(g_{1,2}\left(R, \theta_{\xi}\right)\right)^{2}}{(t-\tau)^{2}}\right] d \theta_{\xi}
\end{aligned}
$$




$$
\begin{aligned}
T_{2}(t)= & \frac{1}{\sqrt{\pi}} \int_{0}^{t} d \tau \int_{\left|\theta_{\xi}\right|<\pi / 2} \rho_{1,2}(\tau) \cos \theta_{\xi} \frac{\left(g_{1,2}\left(R, \theta_{\xi}\right)\right)^{3}}{(t-\tau)^{4}}\left[-\frac{\left(g_{1,2}\left(R, \theta_{\xi}\right)\right)^{2}}{(t-\tau)^{2}}+\frac{3}{2}\right] \\
& \times \exp \left[-\frac{\left(g_{1,2}\left(R, \theta_{\xi}\right)\right)^{2}}{(t-\tau)^{2}}\right] d \theta_{\xi}+\frac{1}{\sqrt{\pi}} \int_{0}^{t} d \tau \int_{\left|\theta_{\xi}\right|<\varphi_{m}} U_{1}(\tau) \cos \theta_{\xi} \frac{\left(g_{1}\left(R, \theta_{\xi}\right)\right)^{4}}{(t-\tau)^{5}} \\
& \times \sqrt{1-R^{2} \sin ^{2} \theta_{\xi}}\left[-\frac{2\left(g_{1}\left(R, \theta_{\xi}\right)\right)^{2}}{(t-\tau)^{2}}+3\right] \exp \left[-\frac{\left(g_{1}\left(R, \theta_{\xi}\right)\right)^{2}}{(t-\tau)^{2}}\right] d \theta_{\xi} \\
& +\frac{1}{\sqrt{\pi}} \int_{0}^{t} d \tau \int_{\left|\theta_{\xi}\right|<\pi / 2} T_{1,2}(\tau) \cos \theta_{\xi} \frac{\left(g_{1,2}\left(R, \theta_{\xi}\right)\right)^{3}}{(t-\tau)^{4}}\left[-\frac{\left(g_{1,2}\left(R, \theta_{\xi}\right)\right)^{4}}{(t-\tau)^{4}}+\frac{5\left(g_{1,2}\left(R, \theta_{\xi}\right)\right)^{2}}{2(t-\tau)^{2}}-2\right] \\
& \times \exp \left[-\frac{\left(g_{1,2}\left(R, \theta_{\xi}\right)\right)^{2}}{(t-\tau)^{2}}\right] d \theta_{\xi},
\end{aligned}
$$

where $\varphi_{m}=\sin ^{-1}(1 / R)$. Equations (25)-(28) are subject to the initial conditions

$$
\rho_{1}(0)=\frac{7 \sqrt{\pi}}{8} U_{1}(0)-\sqrt{\pi} Q_{1}(0), T_{1}(0)=\frac{\sqrt{\pi}}{4} U_{1}(0)+2 \sqrt{\pi} Q_{1}(0), \rho_{2}(0)=0, T_{2}(0)=0
$$

The initial-value problem in Eqs. (25)-(29) may be formulated for arbitrary choices of $U_{1}(t)$ and $Q_{1}(t)$, where the integrations over $\theta_{\xi}$ and $\tau$ are carried out numerically. Once $\rho_{1,2}(t)$ and $T_{1,2}(t)$ are obtained, the perturbation function $\phi$ in Eq. (21) is specified, and the $O(\varepsilon)$ density, radial velocity, temperature, and radial heat-flux perturbations may be computed via the velocity-space quadratures [26]

$$
\begin{aligned}
& \rho(t, r)=\frac{1}{\pi^{3 / 2}} \int_{-\infty}^{\infty} d \xi_{z} \int_{0}^{\infty} d \xi_{2 \mathrm{D}} \int_{-\pi}^{\pi} \phi \xi_{2 \mathrm{D}} \exp \left[-\xi_{2 \mathrm{D}}^{2}-\xi_{z}^{2}\right] d \theta_{\xi}, \\
& u_{r}(t, r)=\frac{1}{\pi^{3 / 2}} \int_{-\infty}^{\infty} d \xi_{z} \int_{0}^{\infty} d \xi_{2 \mathrm{D}} \int_{-\pi}^{\pi} \phi \xi_{2 \mathrm{D}}^{2} \cos \theta_{\xi} \exp \left[-\xi_{2 \mathrm{D}}^{2}-\xi_{z}^{2}\right] d \theta_{\xi}, \\
& T(t, r)=\frac{2}{3 \pi^{3 / 2}} \int_{-\infty}^{\infty} d \xi_{z} \int_{0}^{\infty} d \xi_{2 \mathrm{D}} \int_{-\pi}^{\pi} \phi\left(\xi_{2 \mathrm{D}}^{3}+\xi_{2 \mathrm{D}} \xi_{z}^{2}\right) \exp \left[-\xi_{2 \mathrm{D}}^{2}-\xi_{z}^{2}\right] d \theta_{\xi}-\rho(t, r), \\
& q_{r}(t, r)=\frac{1}{2 \pi^{3 / 2}} \int_{-\infty}^{\infty} d \xi_{z} \int_{0}^{\infty} d \xi_{2 \mathrm{D}} \int_{-\pi}^{\pi} \phi\left(\xi_{2 \mathrm{D}}^{4}+\xi_{2 \mathrm{D}}^{2} \xi_{z}^{2}\right) \cos \theta_{\xi} \exp \left[-\xi_{2 \mathrm{D}}^{2}-\xi_{z}^{2}\right] d \theta_{\xi}-\frac{5}{4} u_{r}(t, r),
\end{aligned}
$$

respectively. The pressure perturbation is obtained by the linearized form of the equation of state,

$$
p(t, r)=[\rho(t, r)+T(t, r)] / 2,
$$

where the $1 / 2$ factor originates from the scaling of pressure by $\rho_{0}^{*} U_{\mathrm{th}}^{2}=2 \rho_{0}^{*} \mathcal{R}^{*} T_{0}^{*}$.

In general, all $\xi_{z}$-integrals appearing in Eq. (30) may be computed in a closed form, while the $\xi_{2 \mathrm{D}}$ and $\theta_{\xi}$ quadratures are carried out numerically, based on the computed $\rho_{1,2}(t)$ and $T_{1,2}(t)$. In the special case of a single cylinder, $\rho_{1,2}(t)$ and $T_{1,2}(t)$ are known in a closed form, and asymptotic evaluation of the hydrodynamic fields may be carried out (see Sec. IV A). The ballistic-flow approximation is expected to hold at distances not exceeding one mean free-path from the boundaries. This becomes relevant when the gap between the cylinders, $\Delta R=R-1$, is of the order of the mean free path, or when the time-scale characterizing walls excitation is of the order of the mean free time. In the latter scenario, equivalent to the condition $\omega \mathrm{Kn} \gtrsim 1$, the effect of wall excitations penetrates less than a mean free path into the gas layer regardless of the distance between the boundaries, as demonstrated in Sec. IV. 


\section{Continuum limit}

In the limit of small Knudsen numbers, we make use of a continuum model, consisting of the Navier-Stokes-Fourier equations, together with the impermeability and heat-flux conditions at the boundaries. Applying the scaling introduced in Sec. II, and linearizing the set of unsteady one-dimensional equations in cylindrical coordinates, we obtain the following $O(\varepsilon)$ balances of mass,

$$
\frac{\partial \rho}{\partial t}+\frac{\partial u_{r}}{\partial r}+\frac{u_{r}}{r}=0
$$

momentum,

$$
\frac{\partial u_{r}}{\partial t}=-\frac{1}{2}\left(\frac{\partial \rho}{\partial r}+\frac{\partial T}{\partial r}\right)+\frac{4}{3} \widetilde{\mathrm{Kn}}\left(\frac{\partial^{2} u_{r}}{\partial r^{2}}+\frac{1}{r} \frac{\partial u_{r}}{\partial r}+\frac{u_{r}}{r^{2}}\right)
$$

and energy,

$$
\frac{\partial T}{\partial t}=(1-\gamma)\left(\frac{\partial u_{r}}{\partial r}+\frac{u_{r}}{r}\right)+\frac{\gamma \widetilde{\mathrm{Kn}}}{\operatorname{Pr}}\left(\frac{\partial^{2} T}{\partial r^{2}}+\frac{1}{r} \frac{\partial T}{\partial r}\right) .
$$

In Eqs. (33) and (34), $\widetilde{\mathrm{Kn}}=v^{*} / U_{\mathrm{th}}^{*} L^{*}$ is the modified Knudsen number, with $v^{*}$ denoting the mean kinematic viscosity of the gas. The modified Knudsen number is related to the Knudsen number via

$$
\widetilde{\mathrm{Kn}}=\frac{v^{*}}{U_{\mathrm{th}}^{*} l^{*}} \mathrm{Kn},
$$

where $v^{*} / U_{\mathrm{th}}^{*} l^{*}=5 \sqrt{\pi} / 16$ for the hard-sphere gas model considered here [26]. Also appearing in Eq. (34) are $\gamma$ and Pr, marking the ratio of specific heats and Prandtl number, respectively. For a perfect monatomic gas, $\gamma=5 / 3$ and $\operatorname{Pr}=2 / 3$. The equations are supplemented by the boundary conditions

$$
u_{r}(r=1, R)=U_{1,2}(t) \text { and }-\left.\alpha \frac{\partial T}{\partial r}\right|_{1, R}=Q_{1,2}(t),
$$

imposing impermeability and specifying the radial heat flux at the walls, respectively. Here, $U_{2}(t)=$ $Q_{2}(t)=0$, as stated in Sec. II, and $\alpha=15 \widetilde{\mathrm{Kn}} / 8$ for a hard-sphere gas [26].

To facilitate the analysis, the problem is solved for the case of harmonic boundary excitation,

$$
U_{1}(t)=\tilde{U}_{1} \cos (\omega t) \text { and } Q_{1}(t)=\tilde{Q}_{1} \cos \left(\omega t+\varphi_{1}\right),
$$

where $\tilde{U}_{1}$ and $\tilde{Q}_{1}$ denote the amplitudes of walls signals, $\omega$ is the frequency of prescribed oscillations, and $\varphi_{1}$ is the phase shift between mechanical and thermal inputs. Having specified the form of excitation, the results presented below may be regarded as the $\omega$-component of the system response to arbitrary time excitation. Assuming a solution of the form

$$
\Phi(t, r)=\operatorname{Re}\{\tilde{\Phi}(r) \exp [i \omega t]\}
$$

for all fields, Eqs. (32)-(34) and (36) are transformed into a system of ordinary equations,

$$
\begin{aligned}
& i \omega \tilde{\rho}+\tilde{u}_{r}^{\prime}+\frac{\tilde{u}_{r}}{r}=0, \quad i \omega \tilde{u}_{r}=\frac{1}{2}\left(\tilde{\rho}^{\prime}+\tilde{T}^{\prime}\right)+\frac{4 \widetilde{\mathrm{Kn}}}{3}\left(\tilde{u}_{r}^{\prime \prime}+\frac{\tilde{u}_{r}^{\prime}}{r}-\frac{\tilde{u}_{r}}{r^{2}}\right), \\
& i \omega \tilde{T}=(1-\gamma)\left(\tilde{u}_{r}^{\prime}+\frac{\tilde{u}_{r}}{r}\right)+\frac{\gamma \widetilde{\mathrm{Kn}}}{\operatorname{Pr}}\left(\tilde{T}^{\prime \prime}+\frac{\tilde{T}^{\prime}}{r}\right),
\end{aligned}
$$

and boundary conditions

$$
\tilde{u}_{r}(r=1)=\tilde{U}_{1}, \quad \tilde{u}_{r}(r=R)=0, \quad-\alpha \tilde{T}^{\prime}(r=1)=\tilde{Q}_{1}, \quad \text { and } \quad-\alpha \tilde{T}^{\prime}(r=R)=0,
$$


where primes denote differentiations with respect to $r$. Eliminating the density and radial velocity via

$$
\tilde{\rho}=\frac{i}{\omega}\left(\tilde{u}_{r}^{\prime}+\frac{\tilde{u}_{r}}{r}\right) \text { and } \quad \tilde{u}_{r}=\frac{i}{\omega(1-\gamma)}\left\{k m \tilde{T}^{\prime \prime \prime}+\frac{k m}{r} \tilde{T}^{\prime \prime}+\left[\frac{1}{2}(1-\gamma)-m\left(i \omega+\frac{k}{r^{2}}\right)\right] \tilde{T}^{\prime}\right\}
$$

yields a single equation for the temperature perturbation,

$$
\begin{gathered}
k m \tilde{T}^{\prime \prime \prime \prime}+\frac{2 k m}{r} \tilde{T}^{\prime \prime \prime}-\left[\frac{k m}{r^{2}}+\frac{1}{2}(\gamma-1)+i \omega(k+m)\right] \tilde{T}^{\prime \prime} \\
+\left[\frac{k m}{r^{3}}+\frac{1}{2 r}(1-\gamma)-\frac{i \omega}{r}(k+m)\right] \tilde{T}^{\prime}-\omega^{2} \tilde{T}=0,
\end{gathered}
$$

subject to the conditions

$$
\begin{aligned}
k m \tilde{T}^{\prime \prime \prime}(1)+k m \tilde{T}^{\prime \prime}(1)-\left[\frac{1}{2}(\gamma-1)+m(i \omega+k)\right] \tilde{T}^{\prime}(1) & =i \omega(\gamma-1) \tilde{U}_{1}, \\
k m \tilde{T}^{\prime \prime \prime}(R)+\frac{k m}{R^{2}} \bar{T}^{\prime \prime}(R)-\left[\frac{1}{2}(\gamma-1)+m\left(i \omega+\frac{k}{R^{2}}\right)\right] \tilde{T}^{\prime}(R) & =0, \\
-\alpha \tilde{T}^{\prime}(1)=\tilde{Q}_{1},-\alpha \tilde{T}^{\prime}(R) & =0 .
\end{aligned}
$$

In Eqs. (41)-(43),

$$
k=\frac{\gamma \widetilde{\mathrm{Kn}}}{\operatorname{Pr}} \text { and } m=\frac{4 \widetilde{\mathrm{Kn}}}{3}-\frac{i}{2 \omega}
$$

Equation (42) may be formed in an invariant notation [27],

$$
k m \nabla^{4} \tilde{T}-\left[\frac{1}{2}(\gamma-1)+i \omega(k+m)\right] \nabla^{2} \tilde{T}-\omega^{2} \tilde{T}=0,
$$

where $\nabla^{4}$ and $\nabla^{2}$ mark the Biharmonic and Laplace operators, respectively. The transformed temperature perturbation is therefore amenable to the closed-form solution

$$
\tilde{T}(r)=a_{1} J_{0}\left(i \sqrt{\lambda_{1}} r\right)+b_{1} Y_{0}\left(i \sqrt{\lambda_{1}} r\right)+a_{2} J_{0}\left(i \sqrt{\lambda_{2}} r\right)+b_{2} Y_{0}\left(i \sqrt{\lambda_{2}} r\right),
$$

where $J_{i}$ and $Y_{i}$ are the $i$ th order Bessel functions of the first and second kind, respectively, and $\lambda_{1}$ and $\lambda_{2}$ are the roots of the characteristic equation

$$
k m \lambda^{2}-\left(\frac{1}{2}(\gamma-1)+i \omega(k+m)\right) \lambda-\omega^{2}=0
$$

given by

$$
\lambda_{1,2}=\frac{1}{2 k m}\left(\frac{1}{2}(\gamma-1)+i \omega(k+m) \pm\left\{\left[\frac{1}{2}(\gamma-1)+i \omega(k+m)\right]^{2}+4 k m \omega^{2}\right\}^{1 / 2}\right) .
$$

The constants of integration $a_{1}, b_{1}, a_{2}$, and $b_{2}$ appearing in Eq. (45) are obtained by imposition of the boundary conditions in Eq. (43). Reverting to the time domain, the temperature perturbation is given by

$$
T(r, t)=\operatorname{Re}\left\{\left[a_{1} J_{0}\left(i \sqrt{\lambda_{1}} r\right)+b_{1} Y_{0}\left(i \sqrt{\lambda_{1}} r\right)+a_{2} J_{0}\left(i \sqrt{\lambda_{2}} r\right)+b_{2} Y_{0}\left(i \sqrt{\lambda_{2}} r\right)\right] \exp [i \omega t]\right\} .
$$

All other perturbation fields are obtained by substitution of Eq. (45) into Eq. (41) and reverting to time using Eq. (38).

The continuum-limit analysis should hold at distances that are much larger than the mean free path from the boundaries and for time-scales that are much longer than the mean free time. In terms of 
the frequency of oscillations, the latter condition holds for $\omega \mathrm{Kn} \ll 1$, or, equivalently, for $\omega \widetilde{\mathrm{Kn}} \ll 1$ [see Eq. (35)]. Examination of this limitation on the approximation is discussed in Sec. IV.

\section{RESULTS}

We start by presenting our results in Sec. IV A for the degenerated case of a single pulsating cylinder $(R \rightarrow \infty)$, to investigate the canonic problem of acoustic radiation in a semiconfined domain. In Sec. IV B, we examine the problem in a confined two-cylinder setup. In each configuration, the cases of an adiabatic pulsating cylinder $\left(\tilde{U}_{1} \neq 0, \tilde{Q}_{1}=0\right)$, and a stationary nonadiabatic cylinder $\left[\tilde{U}_{1}=0, \tilde{Q}_{1} \neq 0\right.$; see Eq. (37)] are considered separately. The combined effects of mechanical and thermal excitations, and their significance in monitoring the acoustic signal, are discussed in Sec. IV C, for a single-cylinder setup.

To quantitate the impact of gas rarefaction on the acoustic disturbance, it is useful to compare our findings with the known result for a cylinder pulsating in an ideal (inviscid and compressible) gas [1]. In this case, the dynamic and thermodynamic fluid descriptions are decoupled, and isothermal conditions prevail. For a single oscillating cylinder, the radial velocity and pressure perturbations are given by

$u_{r}(r, t)=\operatorname{Re}\left\{\tilde{U} \frac{H_{1}^{(1)}(-\omega r \sqrt{2 / \gamma})}{H_{1}^{(1)}(-\omega \sqrt{2 / \gamma})} \exp [i \omega t]\right\}, \quad p(r, t)=\operatorname{Re}\left\{-i \tilde{U} \frac{\sqrt{\gamma} H_{0}^{(1)}(-\omega r \sqrt{2 / \gamma})}{\sqrt{2} H_{1}^{(1)}(-\omega \sqrt{2 / \gamma})} \exp [i \omega t]\right\}$,

whereas for a two-cylinder setup,

$$
\begin{aligned}
u_{r}(r, t) & =\operatorname{Re}\left\{-\sqrt{2 / \gamma}\left[d_{1} J_{1}(-\omega r \sqrt{2 / \gamma})+d_{2} Y_{1}(-\omega r \sqrt{2 / \gamma})\right] \exp [i \omega t]\right\} \\
p(r, t) & =\operatorname{Re}\left\{\left[d_{1} J_{0}(-\omega r \sqrt{2 / \gamma})+d_{2} Y_{0}(-\omega r \sqrt{2 / \gamma})\right] \exp [i \omega t]\right\}
\end{aligned}
$$

In Eq. (49), $H_{i}^{(j)}$ denotes the Hankel function of the $i$ th order and $j$ th kind. In Eq. (50), $d_{1}$ and $d_{2}$ are determined by walls impermeability conditions. The $\sqrt{2 / \gamma}$ factor appearing in Eqs. (49) and (50) expresses the ratio between the mean thermal speed, used for the scaling of velocity (see Sec. II), and the mean speed of sound.

\section{A. The case of a single cylinder}

\section{Ballistic-flow limit}

Having removed the outer cylinder, no boundary reflections occur at $r=R$, and the system description simplifies considerably. Setting $\rho_{2}(t)=T_{2}(t) \equiv 0$ in Eqs. (25) and (26), we find

$$
\rho_{1}(t)=\frac{7 \sqrt{\pi}}{8} U_{1}(t)-\sqrt{\pi} Q_{1}(t) \text { and } T_{1}(t)=\frac{\sqrt{\pi}}{4} U_{1}(t)+2 \sqrt{\pi} Q_{1}(t) .
$$

The probability density perturbation $\phi(r, t, \xi)$ in Eq. (21) is therefore known, and explicit evaluation of the hydrodynamic perturbations in Eqs. (30) and (31) may be carried out. Introducing

$$
\begin{aligned}
I_{n}^{(k)} & =\int_{0}^{\infty} d \xi_{2 \mathrm{D}} \int_{-\varphi}^{\varphi} U_{1}\left(t_{1}\right) f^{(k)}\left(\theta_{\xi}\right) \sqrt{1-r^{2} \sin ^{2}\left(\theta_{\xi}\right)} \xi_{2 \mathrm{D}}^{n} \exp \left[-\xi_{2 \mathrm{D}}^{2}\right] d \theta_{\xi}, \\
J_{n}^{(k)} & =\int_{0}^{\infty} d \xi_{2 \mathrm{D}} \int_{-\varphi}^{\varphi} \rho_{1}\left(t_{1}\right) \xi_{2 \mathrm{D}}^{n} f^{(k)}\left(\theta_{\xi}\right) \exp \left[-\xi_{2 \mathrm{D}}^{2}\right] d \theta_{\xi}, \\
K_{n}^{(k)} & =\int_{0}^{\infty} d \xi_{2 \mathrm{D}} \int_{-\varphi}^{\varphi} T_{1}\left(t_{1}\right) \xi_{2 \mathrm{D}}^{n} f^{(k)}\left(\theta_{\xi}\right) \exp \left[-\xi_{2 \mathrm{D}}^{2}\right] d \theta_{\xi},
\end{aligned}
$$


where $t_{1}=t-g_{1}\left(r, \theta_{\xi}\right) / \xi_{2 \mathrm{D}}$ and

$$
f^{(k)}\left(\theta_{\xi}\right)=\left\{\begin{array}{ll}
1, & k=1 \\
\cos \left(\theta_{\xi}\right), & k=2
\end{array},\right.
$$

Eqs. (30) and (31) for the hydrodynamic perturbations are cast in the form

$$
\begin{aligned}
& \rho(t, r)=\frac{1}{\pi}\left(2 I_{2}^{(1)}+J_{1}^{(1)}-K_{1}^{(1)}+K_{3}^{(1)}\right), \\
& u(t, r)=\frac{1}{\pi}\left(2 I_{3}^{(2)}+J_{2}^{(2)}-K_{2}^{(2)}+K_{4}^{(2)}\right), \\
& T(t, r)=\frac{2}{3 \pi}\left(-2 I_{2}^{(1)}+2 I_{4}^{(1)}-J_{1}^{(1)}+J_{3}^{(1)}+K_{1}^{(1)}-2 K_{3}^{(1)}+K_{5}^{(1)}\right), \\
& p(t, r)=\frac{1}{3 \pi}\left(I_{2}^{(1)}+2 I_{4}^{(1)}+\frac{1}{2} J_{1}^{(1)}+J_{3}^{(1)}-\frac{1}{2} K_{3}^{(1)}+K_{5}^{(1)}\right), \\
& q(t, r)=\frac{1}{2 \pi}\left(-2 I_{3}^{(2)}+2 I_{5}^{(2)}-2 J_{2}^{(2)}+J_{4}^{(2)}+\frac{5}{2} K_{2}^{(2)}-3 K_{4}^{(2)}+K_{6}^{(2)}\right) .
\end{aligned}
$$

Focusing on the final time-periodic state of the system, Eqs. (52) for $J_{n}^{(1)}$ and $K_{n}^{(1)}$ contain a double integral of the form

$$
G_{n}=\operatorname{Re}\left\{\frac{1}{\pi} \exp [i \omega t] \int_{0}^{\infty} d \xi_{2 \mathrm{D}} \int_{-\varphi}^{\varphi} \xi_{2 \mathrm{D}}^{n} \exp \left[-i \omega g_{1}\left(r, \theta_{\xi}\right) / \xi_{2 \mathrm{D}}-\xi_{2 \mathrm{D}}^{2}\right] d \theta_{\xi}\right\} .
$$

Following Abramowitz [28] to evaluate the $\xi_{2 \mathrm{D}}$-integral, and making use of the method of stationary phase to approximate the $\theta_{\xi}$-integral, we find

$$
G_{n} \approx \operatorname{Re}\left\{\mathbb{C}(r) \sqrt{\frac{z^{n-1}}{3^{n}}}\left(1+\frac{a_{1}}{z}+\frac{a_{2}}{z^{2}}\right) \exp [i \omega t-z]\right\},
$$

valid for $\omega(r-1) \gg 1$, where

$$
\mathbb{C}(r)=\frac{1}{\sqrt{r}} \operatorname{erf}\left[\sin ^{-1}\left(\frac{1}{r}\right) \sqrt{\frac{1}{3} r z}\right],
$$

and $z=3[\omega(r-1) / 2]^{2 / 3} \exp [i \pi / 3]$. The expressions for the constants $a_{1}$ and $a_{2}$ and details on the calculation are given in the Appendix. Since the point of stationary phase occurs at $\theta_{\xi}=0$, Eq. (55) is also valid as a leading-order approximation for $I_{n}^{(1,2)}, J_{n}^{(2)}$, and $K_{n}^{(2)}$ in Eq. (52).

Considering Eqs. (52), (53), and (55), we observe that all the hydrodynamic fields at ballistic-flow conditions decay as $\sim \mathbb{C}(r)[\omega(r-1)]^{(n+1) / 3} \exp \left[-(\omega(r-1))^{2 / 3}\right]$ away from the cylindrical source. The $[\omega(r-1)]^{(n+1) / 3} \exp \left\{-[\omega(r-1)]^{2 / 3}\right\}$ part is identical with the decay rate in the counterpart planar setup [15], and reflects the dampening effect of gas rarefaction. The added $\mathbb{C}(r)$ multiplier reflects the effect of boundary curvature, which magnifies the rate of decay compared with the planar case. Recalling the microscopic flow description discussed in Sec. III B, this increased decay factor is attributed to the narrowing in the $\theta_{\xi}$-section imposed, at a given $r$, on the molecules reflected from the $r=1$ boundary (see Fig. 1 et seq.). Consequently, the number of reflected particles that reach a certain distance from the wall (and thus contribute the far acoustic field) is "geometrically reduced," resulting in a stronger signal decay compared with the planar setup. Notably, the curvature-related "near-field" decay is also significantly stronger than the characteristic $r^{-1 / 2}$ decay in two-dimensional sources at continuum-flow conditions [1] (see Sec. IV A 2), thus manifesting the unique impact of boundary curvature on source acoustic efficiency at noncontinuum conditions. 


\section{Continuum limit}

Considering the continuum limit of small Knudsen numbers, and focusing on large distances from the oscillating cylinder, we observe that, in Eq. (48) for $T(r, t)$,

$$
J_{0}\left(i \sqrt{\lambda_{1,2}} r\right) \sim r^{-1 / 2} \cos \left(i \sqrt{\lambda_{1,2}} r-\pi / 4\right) \text { and } Y_{0}\left(i \sqrt{\lambda_{1,2}} r\right) \sim r^{-1 / 2} \sin \left(i \sqrt{\lambda_{1,2}} r-\pi / 4\right) .
$$

Expecting a causal far-field solution that propagates in the positive $r$-direction, we set $b_{1}=i a_{1}$ and $b_{2}=i a_{2}$ in Eq. (48), to obtain

$$
T(r, t)=\operatorname{Re}\left\{\left[a_{1} H_{0}^{(1)}\left(i \sqrt{\lambda_{1}} r\right)+a_{2} H_{0}^{(1)}\left(i \sqrt{\lambda_{2}} r\right)\right] \exp [i \omega t]\right\},
$$

where $a_{1}$ and $a_{2}$ are fixed via the impermeability and heat-flux conditions at $r=1$. Expanding Eq. (47) for $\lambda_{1,2}$ in the limit $\widetilde{\mathrm{Kn}} \ll 1$ and $\omega \widetilde{\mathrm{Kn}} \ll 1$ of near-continuum conditions, we find

$$
\sqrt{\lambda_{1}} \approx \sqrt{\frac{1}{2} \operatorname{Pr} \frac{\omega}{\widetilde{K n}}}(1+i), \quad \sqrt{\lambda_{2}} \approx \frac{1}{\sqrt{2 \gamma^{3}}}\left(2 \frac{(\gamma-1)}{\operatorname{Pr}}+\frac{8}{3}\right) \omega^{2} \widetilde{\mathrm{Kn}}+i \sqrt{\frac{2}{\gamma}} \omega .
$$

Substituting Eq. (58) into Eq. (57), the propagation velocities of the $\sqrt{\lambda_{1}}$ and $\sqrt{\lambda_{2}}$ waves are

$$
c_{1}=\sqrt{\frac{2}{\operatorname{Pr}} \omega \widetilde{\mathrm{Kn}}} \text { and } c_{2}=\sqrt{\frac{\gamma}{2}},
$$

respectively, and their corresponding decay rates are

$$
\begin{aligned}
& \mathcal{D}_{1} \sim\left(\sqrt{\frac{1}{2} \operatorname{Pr} \frac{\omega}{\widetilde{\mathrm{Kn}}}} r\right)^{-1 / 2} \exp \left[-\sqrt{\frac{1}{2} \operatorname{Pr} \frac{\omega}{\widetilde{\mathrm{Kn}}} r}\right] \text { and } \\
& \mathcal{D}_{2} \sim\left(\sqrt{\frac{2}{\gamma} \omega r}\right)^{-1 / 2} \exp \left[-\frac{\omega^{2} \widetilde{\mathrm{Kn}}}{\sqrt{2 \gamma^{3}}}\left(2 \frac{(\gamma-1)}{\operatorname{Pr}}+\frac{8}{3}\right) r\right] .
\end{aligned}
$$

The $\sqrt{\lambda_{2}}$ wave therefore propagates with the speed of sound $c_{2}\left(\gg c_{1}\right)$. Comparing between $\mathcal{D}_{1}$ and $\mathcal{D}_{2}$, we observe that the $\sqrt{\lambda_{1}}$ decay rate is significantly larger than $\sqrt{\lambda_{2}}$ since $\sqrt{\omega / \widetilde{\mathrm{Kn}}} \gg \omega^{2} \widetilde{\mathrm{Kn}}$. Focusing on the $\widetilde{\mathrm{Kn}} \rightarrow 0$ limit and keeping $\omega$ constant, the above results therefore indicate that the $\sqrt{\lambda_{1}}$ wave vanishes and the flow field is dominated entirely by the $\sqrt{\lambda_{2}}$ wave, with a decay rate of $\mathcal{D}_{2} \sim(\omega r \sqrt{2 / \gamma})^{-1 / 2}$. In this limit, the dynamical problem turns decoupled from the thermal description, and the system response at inviscid and isothermal conditions is recaptured [1]. For later convenience, we refer to the $\sqrt{\lambda_{1}}$ and $\sqrt{\lambda_{2}}$ waves as the "thermal" and "compression" waves, respectively. These terms have also been used in Ref. [29] and earlier works, to analyze the acoustic scattering by cylinders and spheres interacting with plane sound waves at continuum conditions.

\section{Numerical results}

To validate the collisionless- and continuum-limit predictions in Secs. IV A 1 and IV A 2, Fig. 2 presents comparison between the analytical and DSMC results for the acoustic pressure at fixed $\omega=3 \pi / 2$. The responses to both adiabatic $\left[\tilde{U}_{1}=0.01, \tilde{Q}_{1}=0\right.$; Fig. $2($ a) $]$ and thermal $\left[\tilde{U}_{1}=0\right.$, $\tilde{Q}_{1}=0.01$; Fig. 2(b)] excitations are illustrated at time $t=2 \pi n / \omega$. In both figures, the collisionless results are compared with $\mathrm{Kn}=5 \mathrm{DSMC}$ predictions, and the continuum-limit and DSMC calculated fields are compared for $\mathrm{Kn}=0.03$. In Fig. 2(a), the inviscid $\mathrm{Kn} \rightarrow 0$ response in Eq. (49) is added for reference. A counterpart result in Fig. 2(b) is inevitably missing, as the dynamic and thermodynamic responses are decoupled in this limit.

Comparing between the $\mathrm{Kn}=0.03$ and $\mathrm{Kn} \rightarrow 0$ results in Fig. 2(a), the thermoviscous effect on wave decay is apparent, reducing the width of the layer affected by the acoustic signal with increasing $\mathrm{Kn}$. This supports the discussion in Sec. IV A 2, showing that the system response in the continuum limit, dominated by the compression wave, is characterized by a product of "potential" inverse 

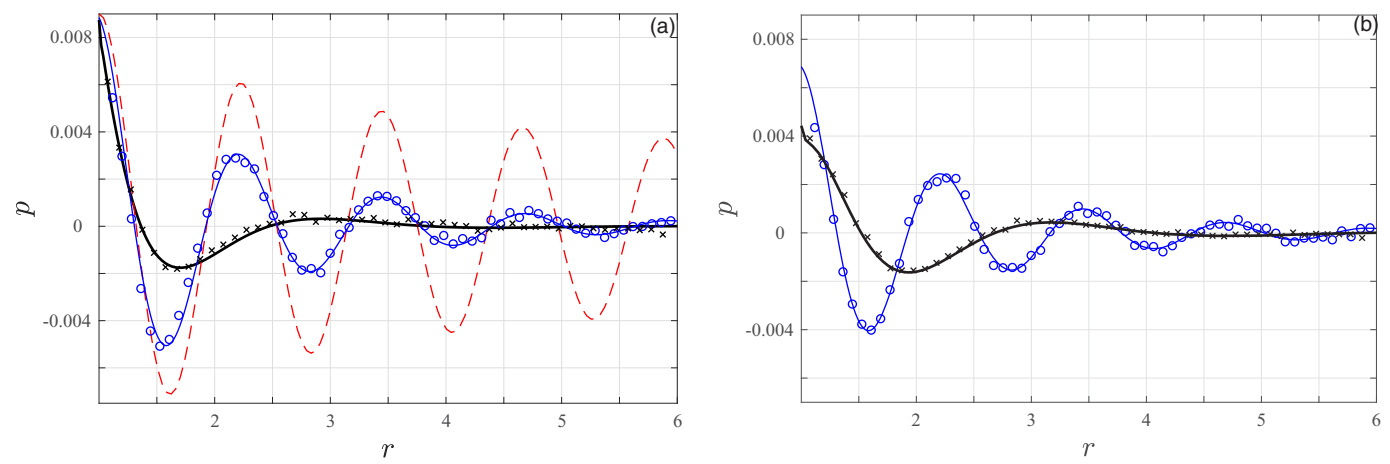

FIG. 2. Effect of the Knudsen number on the pressure perturbation in a single-cylinder setup for $\omega=3 \pi / 2$ : (a) adiabatic animation $\left(\tilde{U}_{1}=0.01, \tilde{Q}_{1}=0\right)$, (b) heat-flux forcing $\left(\tilde{U}_{1}=0, \tilde{Q}_{1}=0.01\right)$. The thick solid lines, thin blue lines and dashed red curve present analytical predictions for collisionless, $\mathrm{Kn}=0.03$ and inviscid $(\mathrm{Kn} \rightarrow 0)$ gas, respectively, and the crosses and blue circles mark DSMC data for $\mathrm{Kn}=5$ and $\mathrm{Kn}=0.03$, respectively. The results are presented at time $t=2 \pi n / \omega$.

square root $\left(\propto r^{-1 / 2}\right)$ and viscous exponential $\left(\propto \exp \left[-\omega^{2} \mathrm{Kn}\right]\right)$ decay factors. At the other extreme of free-molecular flow, signal decay becomes ever stronger, with the layer affected by boundary excitations confined to only few $(\lesssim 3)$ radii into the gas, in line with the discussion in Sec. IV A 1.

Inspecting the differences between the analytical and DSMC predictions, and in view of the vastly different schemes of solution, we find that the agreement between the results is gratifying. Any discrepancies observed are exceedingly small and may be attributed to the statistical noise inherent in the Monte Carlo calculations. The analytical solutions may therefore be considered as a viable means for predicting the system behavior at the two limits. This becomes particularly useful in the continuum limit, where the signal affects a wide layer away from the wall, and DSMC calculations become prohibitively time consuming.

Figure 3 describes the effect of excitation frequency $\omega$ on the system response. The figure shows the pressure perturbation for the cases of adiabatic pulsation [Figs. 3(a) and 3(c)] and thermal excitation [Figs. 3(b) and 3(d)] with $\omega=3 \pi / 2$ and $\omega=3 \pi / 4$. Both large- and small-Kn number limits are presented and compared with DSMC data. With the higher frequency being twice the lower, the results confirm that the wavelength of the disturbance in the latter case is doubled. Notably, the impact of $\omega$ on the decay rate varies between the limits, with a considerable attenuating effect observed at continuum conditions. The dampening effect of $\omega$ is supported by Eq. (60) for $\mathcal{D}_{2}$, showing an $(\omega r)^{-1 / 2} \exp \left[-\omega^{2} \mathrm{Kn} r\right]$ dependence. This $\omega^{2}$-exponential decay changes at collisionless conditions, where Eq. (55) predicts a milder $\omega$-dependence of the $r$-attenuation $\propto$ $\exp \left\{-[\omega(r-1)]^{2 / 3}\right\}$. The results are well supported by DSMC predictions.

Having demonstrated cases where the limit-case analyses agree with DSMC results, it is of interest to examine the conditions where these descriptions break down. Toward this end, Fig. 4 compares the pressure perturbation between the different schemes at various values of $\omega$ and relatively large [Figs. 4(a) and 4(b)] and small [Fig. 4(c)] Kn. Considering the high-Kn comparison in Fig. 4(a), we observe that, for $\omega=3 \pi / 2$, the collisionless and $\mathrm{Kn}=5$ results agree well, while increasing deviations appear for $\mathrm{Kn}=0.5$ and $\mathrm{Kn}=0.1$. These deviations, reflecting the effect of molecular collisions, become vanishingly small at the higher $\omega=15 \pi$ frequency presented in Fig. 4(b), for which the affected gas layer is thinner. It is therefore the product $\omega \mathrm{Kn}$, denoting the scaled actuation frequency in mean-collision-frequency units [see Eq. (3) and the scaling introduced in Sec. II], which determines the applicability of collisionless conditions, requiring that $\omega \mathrm{Kn} \gg 1$. Passing to the low-Kn results in Fig. 4(c), it is observed that $\omega \mathrm{Kn}$ also governs the validity of the continuum-limit description, now requiring $\omega \mathrm{Kn} \ll 1$. Specifically, good matching between DSMC and analytical results is achieved for $\mathrm{Kn}=0.05$ and $\omega=3 \pi / 4$, where $\omega \mathrm{Kn} \approx 0.12$, while nonsmall deviations are observed for $\mathrm{Kn}=0.1$ and $\omega=3 \pi / 2$, where $\omega \mathrm{Kn} \approx 0.47$. Identifying $\omega \mathrm{Kn}$ as the parameter 

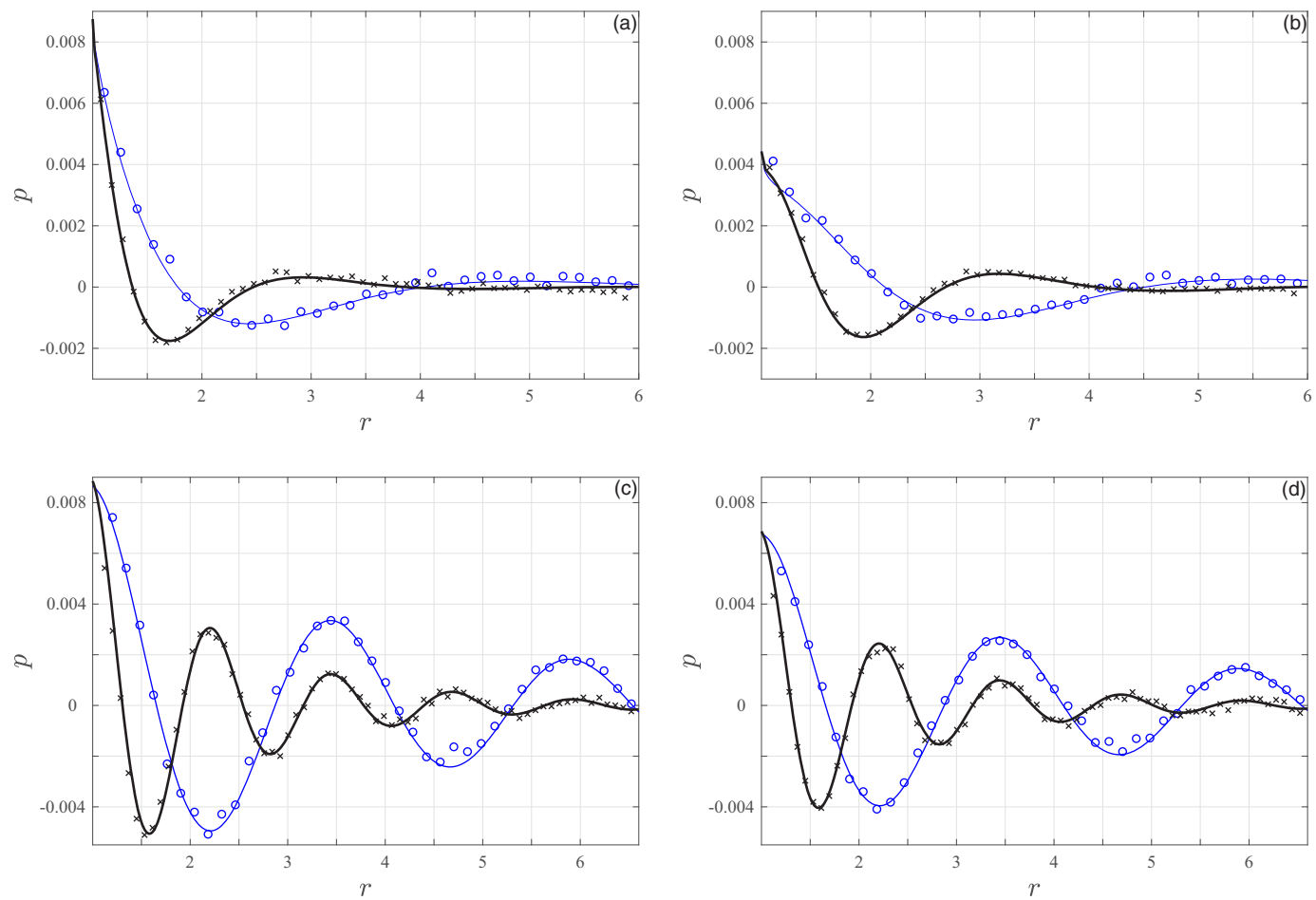

FIG. 3. Effect of the excitation frequency on the pressure perturbation in a single-cylinder setup in response to (a), (c) adiabatic animation $\left(\tilde{U}_{1}=0.01, \tilde{Q}_{1}=0\right)$, (b), (d) heat-flux forcing $\left(\tilde{U}_{1}=0, \tilde{Q}_{1}=0.01\right)$. In (a), (b), the thick and blue thin solid curves present collisionless predictions for $\omega=3 \pi / 2$ and $\omega=3 \pi / 4$, respectively, and the crosses and blue circles mark respective DSMC data for $\mathrm{Kn}=5$. In (c), (d), the thick and blue thin lines present continuum-limit predictions for $\mathrm{Kn}=0.03$ with $\omega=3 \pi / 2$ and $\omega=3 \pi / 4$, respectively, and the crosses and blue circles mark respective DSMC data. The results are presented at time $t=2 \pi n / \omega$.

governing the flow regime may be physically rationalized, since no "natural" lengthscale exists in the unconfined single-cylinder setup, and it is the ratio between the actuation time scale, $1 / \omega^{*}$, and the microscopic time scale, $l^{*} / U_{\mathrm{th}}^{*}$, that determines the flow conditions. This situation changes in the double-cylinder setup, as discussed in Sec. IV B.

\section{B. Double-cylinder setup}

We now discuss the acoustic field generated in the confined double-cylinder setup. Having an outer cylinder, the gap between the cylinders,

$$
\Delta R=R-1,
$$

is introduced as an additional geometrical length scale. This affects both the flow-field (via gas reflections at the outer wall) and applicability of the limit-case approximations, as discussed below.

Fixing $\Delta R=1$ (i.e., taking a gap equal to the inner-cylinder radius), Fig. 5 presents the effect of the Knudsen number on the acoustic pressure and radial velocity for both adiabatic animation and thermal excitation of the inner wall with $\omega=3 \pi / 2$. DSMC and counterpart collisionless and continuum-limit solutions are compared for large $(\mathrm{Kn}=5)$ and small $(\mathrm{Kn}=0.01)$ Knudsen numbers. The reference continuum inviscid $(\mathrm{Kn} \rightarrow 0)$ solution for the adiabatic case is shown in Figs. 5(a) and 5(c). The agreement between DSMC and analytic results is satisfactory in all cases, reconfirming the validity of our calculations. The results for the radial velocity also confirm the satisfaction of the impermeability condition at both walls. 

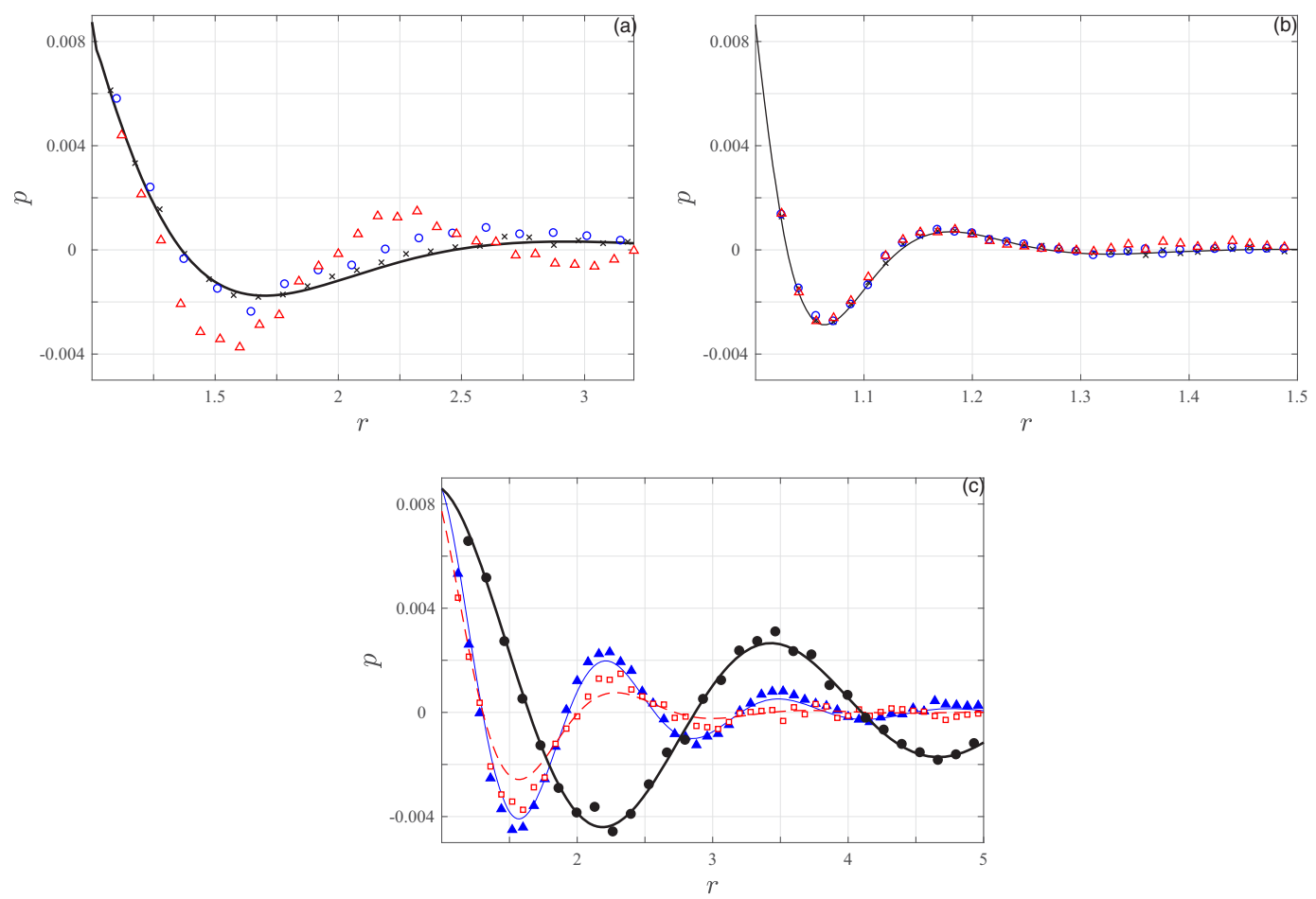

FIG. 4. Breakdown of the (a), (b) collisionless and (c) continuum descriptions in a single-cylinder setup for the case of adiabatic boundary excitation $\left(\tilde{U}_{1}=0.01, \tilde{Q}_{1}=0\right)$, depicted by the pressure perturbation. (a), (b) Comparison between collisionless-limit results (solid line) and DSMC data $(\mathrm{Kn}=5$, crosses; $\mathrm{Kn}=0.5$, blue circles; $\mathrm{Kn}=0.1$, red triangles) for (a) $\omega=3 \pi / 2$ and (b) $\omega=15 \pi$. (c) Comparison between continuum-limit predictions and DSMC data for $\omega=3 \pi / 2$ and $\mathrm{Kn}=0.05$ (blue thin line vs. full blue triangles); $\omega=3 \pi / 2$ and $\mathrm{Kn}=0.1$ (red dashed curve vs. red squares); and $\omega=3 \pi / 4$ and $\mathrm{Kn}=0.05$ (thick line vs. full circles). The results are presented at time $t=2 \pi n / \omega$.

Comparing between the $\mathrm{Kn}=0.01$ and $\mathrm{Kn} \rightarrow 0$ results in Figs. 5(a) and 5(c), we find that the effect of gas rarefaction on the hydrodynamic field at small (yet nonzero) $\mathrm{Kn}$ is vanishingly small. As recalled from the discussion in Sec. IV A, the impact of the deviation from continuum conditions at $\mathrm{Kn} \ll 1$ in a single-cylinder setup is expressed by a thermoviscous decay factor, formed as an exponential $\exp \left[-\omega^{2} \mathrm{Kn} r\right]$ term. While this difference becomes significant away from the wall [cf. the thin and dashed curves in Fig. 2(a)], its influence in a confined geometry should be smaller, as confirmed by the present comparison. Clearly, the added wall may impact the signal only in cases where it is placed at a distance shorter than the "penetration layer" affected by the boundary in the counterpart single-cylinder setup. Thus, observing the results in Fig. 2 for $\omega=3 \pi / 2$, reducing the gap below $\Delta R \approx 4$ and $\Delta R \approx 10$ at free-molecular and $\mathrm{Kn}=0.03$ conditions, respectively, should increasingly modify the resulting flow field.

By adding the outer wall and consequent gap-width length scale, the gap-width-based Knudsen number,

$$
\mathrm{Kn}_{\Delta R}=\frac{l^{*}}{\Delta R^{*}}=\frac{\mathrm{Kn}}{\Delta R},
$$

is introduced. This adds limitations to the range of applicability of the limit-case solutions, compared with the single-cylinder case discussed in Fig. 4. To observe that, Fig. 6 presents the effect of the gap width $\Delta R$ on the acoustic pressure and radial velocity excited by adiabatic pulsations at $\omega=3 \pi / 2$ and relatively large [Fig. 6(a)] and low [Fig. 6(b)] Knudsen numbers. Comparing the results in 

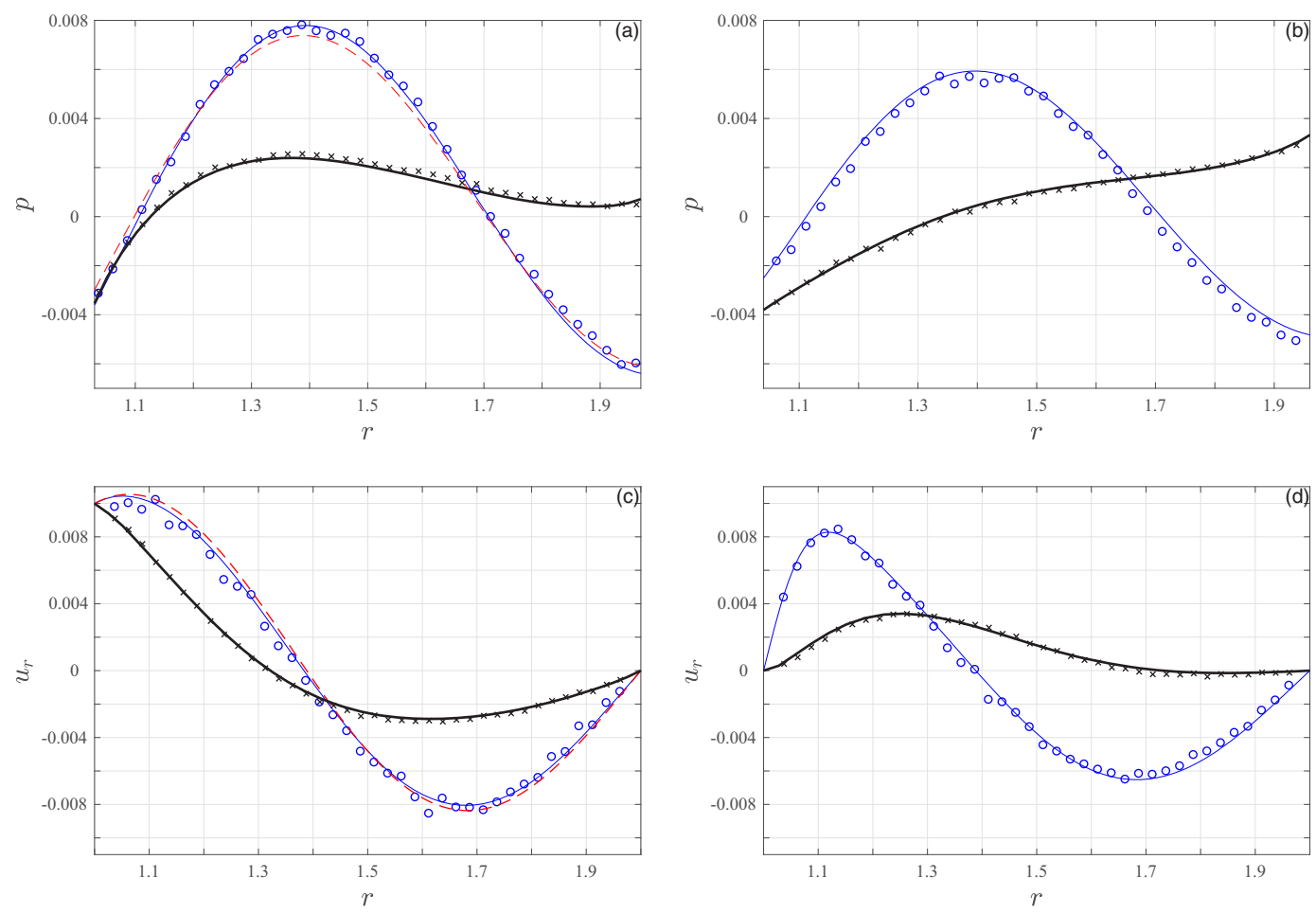

FIG. 5. Effect of the Knudsen number on the (a), (b) pressure and (c), (d) radial velocity perturbations in a double-cylinder setup with $\omega=3 \pi / 2$ : (a), (c) adiabatic animation $\left(\tilde{U}_{1}=0.01, \tilde{Q}_{1}=0\right)$, (b), (d) heat-flux forcing $\left(\tilde{U}_{1}=0, \tilde{Q}_{1}=0.01\right)$. The thick solid lines, thin blue lines, and dashed red curves present analytical predictions for collisionless, $\mathrm{Kn}=0.01$, and inviscid $(\mathrm{Kn} \rightarrow 0)$ gas, respectively, and the crosses and blue circles mark DSMC data for $\mathrm{Kn}=5$ and $\mathrm{Kn}=0.01$, respectively. The results are presented at time $t=$ $2 \pi(n+1 / 3) / \omega$ for the acoustic pressure and at time $t=2 \pi n / \omega$ for the radial velocity field.

Fig. 6(a) with the counterpart single-cylinder results in Fig. 4(a), we observe that the agreement between the $\mathrm{Kn}=0.5$ and free-molecular predictions is much improved in the double-cylinder setup. Thus, it is the increased value of $\mathrm{Kn}_{\Delta R}=5$ (in spite of the relatively non-large value of $\omega \mathrm{Kn}$ ) that diminishes the effect of molecular collisions compared with the nonconfined case, for which the wall effect extends to $r \approx 4$. For the $\mathrm{Kn}=0.01$ and $\Delta R=0.5$ results presented in Fig. 6(b), we find that the agreement is less satisfactory than in the $\Delta R=1$ case shown in Figs. 5(a) and 5(c). Here, gap narrowing and consequent increase in $\mathrm{Kn}_{\Delta R}$ result in the breakdown of the continuum description, even for a relatively small value of $\omega \mathrm{Kn}$.

Having studied the breakdown of the limit-case solutions in the single- and double-cylinder setups, it is concluded that, while $\omega \mathrm{Kn}$ is the dominant parameter governing the flow regime in the former case, it is the combination of $\omega \mathrm{Kn}$ and $\mathrm{Kn}_{\Delta R}$ that governs the flow characteristics in the latter. Since none of the limit-case solutions, including in the single-cylinder case, may be written as a function of $\omega \mathrm{Kn}$ only, it is clear that the separate effects of $\mathrm{Kn}, \omega$, and $\Delta R$ are generally independent. In the one-cylinder case, this particularly reflects the impact of boundary curvature, which turns dominant in the vicinity of the excited cylinder.

\section{Combined animation and thermal excitations}

Having investigated the acoustic pressure generated by a vibrating cylinder and a thermally excited cylinder in separate, it is of interest to consider the system response to a combined vibrothermal 

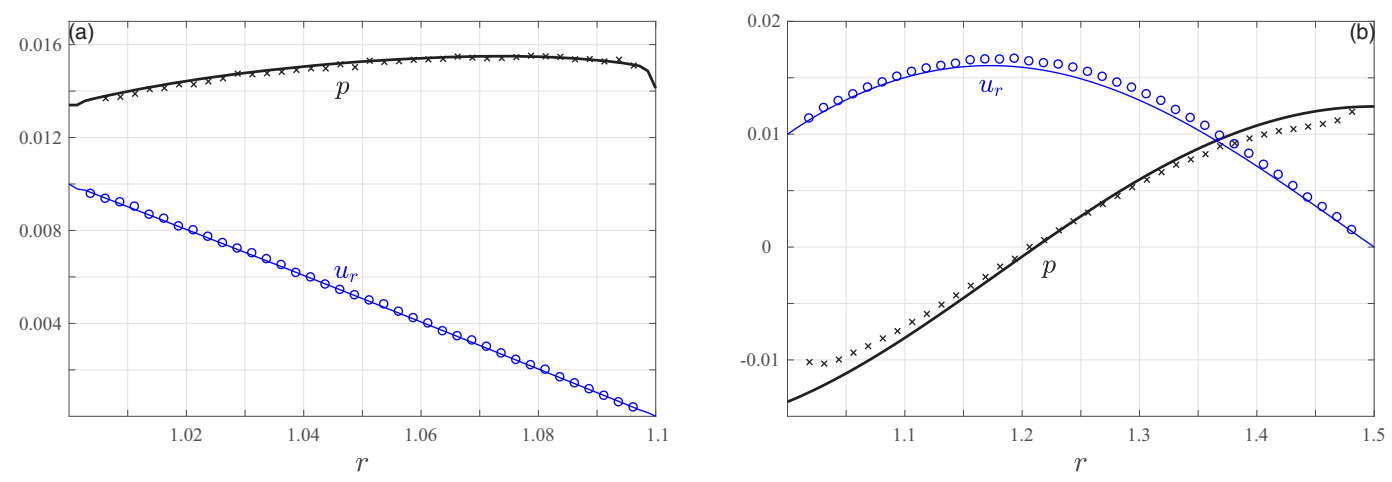

FIG. 6. Effect of the gap width $\Delta R$ on the (a) large-Kn and (b) small-Kn acoustic pressure and radial velocity for the case of adiabatic boundary excitation $\left(\tilde{U}_{1}=0.01, \tilde{Q}_{1}=0\right)$ with $\omega=3 \pi / 2$. (a) Comparison between collisionless (thick and blue thin lines) and DSMC results for $\mathrm{Kn}=0.5$ (crosses and blue circles) with $\Delta R=0.1$. (b) Comparison between continuum-limit (thick and blue thin lines) and DSMC (crosses and blue circles) predictions for $\mathrm{Kn}=0.01$ and $\Delta R=0.5$. The results are presented at time $t=2 \pi n / \omega$ for the velocity and $t=2 \pi(n+1 / 3) / \omega$ for the pressure.

signal. Specifically, we aim at identifying the optimal ratio between heat-flux and normal velocity amplitudes, $\tilde{Q}_{1} / \tilde{U}_{1}$, that reduces the counterpart vibroacoustic signal. For simplicity, we focus on a single-cylinder setup. Our numerical results indicate that an opposite-phase acting heat signal is most efficient for sound attenuation in this case, and we therefore fix $\varphi_{1}=\pi$ in Eq. (37).

Inspecting the system response to a combined vibrothermal input, qualitative differences appear between the continuum and free-molecular gas behaviors. Starting with the small-Kn response, the results obtained agree with previous findings in the planar setup [15] — stating that, based on thermodynamics first-law considerations, optimal sound cancellation is obtained when

$$
\left|\frac{\tilde{Q}_{1}}{\tilde{U}_{1}}\right|_{\omega \mathrm{Kn} \ll 1}^{\mathrm{opt}}=\frac{\gamma}{2(\gamma-1)}=\frac{5}{4} .
$$

This result, valid at near-equilibrium conditions, is found independent of neither the frequency of excitation (as long as $\omega \mathrm{Kn} \ll 1$ ), nor boundary shape. The result may be easily verified by taking the continuum-limit responses presented in Figs. 3(c) and 3(d), multiplying the latter by the required $\gamma /[2(\gamma-1)]$ ratio and subtracting from the former. The subtraction reflects the above-noted opposite-phase difference required between the signals, and imposing wall cooling when the cylinder expands into the gas layer, and vice versa.

To examine the system response at free-molecular conditions to a vibrothermal input, Fig. 7(a) presents the variation with the radial distance of the optimal ratio $\left|\tilde{Q}_{1} / \tilde{U}_{1}\right|^{\text {opt }}$ found for sound minimization. The plot is repeated in Fig. 7(b), where the radial-distance abscissa is replaced by the acoustic wavelength coordinate $\omega(r-1)$. To measure the "quality" of the minimum pressure obtained, Fig. 7(c) shows the variation with $\omega(r-1)$ of the ratio $|p|_{\min } /|p|_{\text {adiab }}$ between the minimum pressure amplitude [at the conditions specified in Fig. 7(b)] and the pressure amplitude in the counterpart adiabatic case $\left(\tilde{U}_{1}=0.01, \tilde{Q}_{1}=0\right)$. The results are plotted for different values of $\omega$, and the uniform optimal $\gamma /[2(\gamma-1)]$ value at continuum-limit conditions is plotted in Fig. $7(\mathrm{~b})$.

Starting with Fig. $7\left(\right.$ a), we observe that the optimal $\left|\tilde{Q}_{1} / \tilde{U}_{1}\right|$ ratio for sound minimization decreases with both $r$ and $\omega$. Notably, when transferring to the $\omega(r-1)$ coordinate in Fig. 7(b), the $\omega$-dependence becomes nearly indistinguishable, suggesting that the optimal ratio essentially depends on the acoustic-wavelength-scaled distance only. This result is nevertheless different from the uniform (in both $r$ and $\omega$ ) continuum-limit value found at near-equilibrium conditions. 

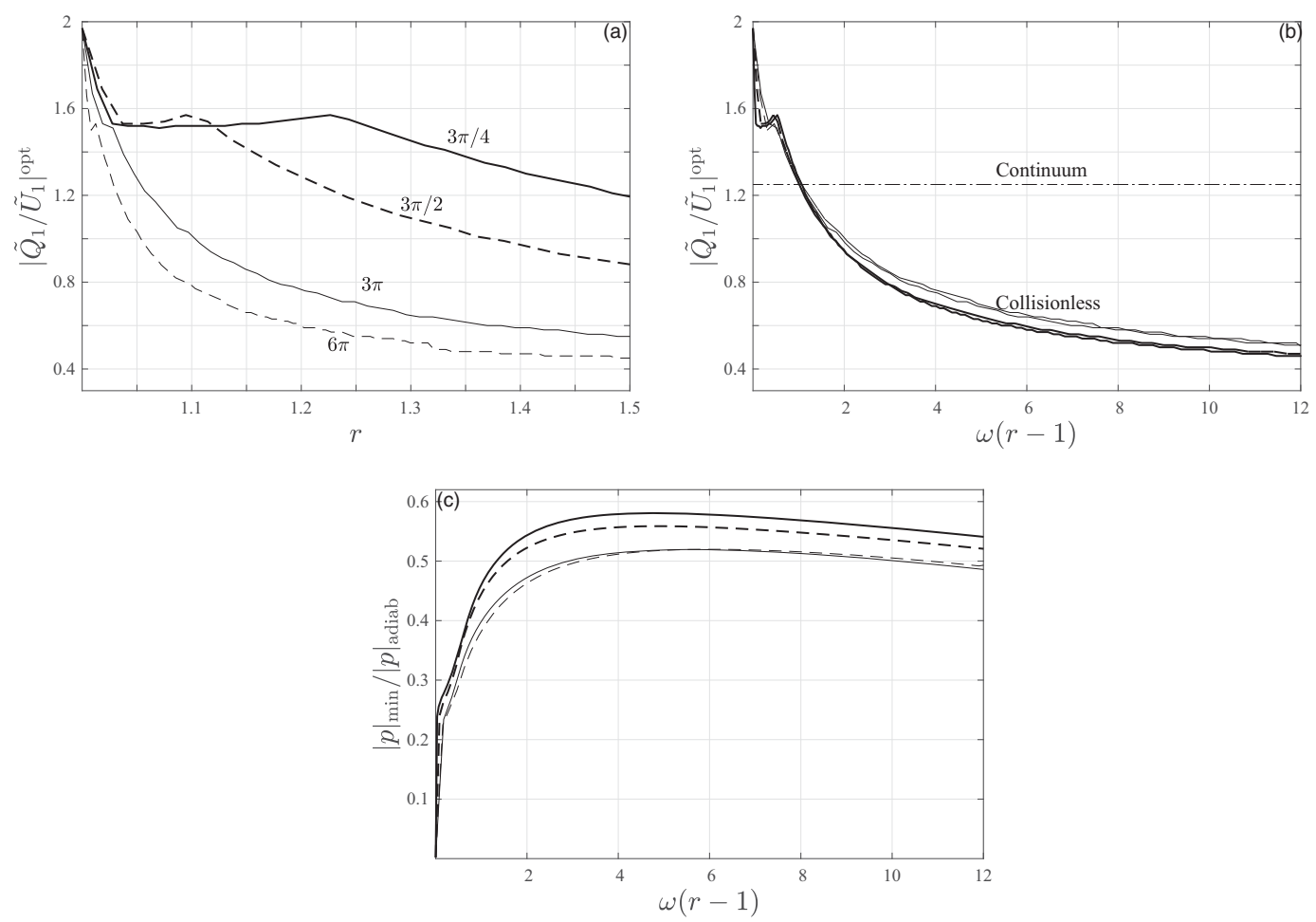

FIG. 7. Combined effects of boundary animation and heating on the acoustic radiation in a single-cylinder setup. (a), (b) Variation with (a) $r$ and (b) $\omega(r-1)$ of the optimal ratio of excitation amplitudes, $\left|\tilde{Q}_{1} / \tilde{U}_{1}\right|^{\text {opt }}$, which minimizes the pressure fluctuation at collisionless-flow conditions. The thick, dashed thick, thin, and dashed thin curves present results for $\omega=3 \pi / 4,3 \pi / 2,3 \pi$, and $6 \pi$, respectively. In Fig. 7(b), the dashdotted curve presents the (uniform) optimal ratio $\gamma /[2(\gamma-1)]=5 / 4$ obtained at continuum-flow conditions. (c) Variation with $\omega(r-1)$ of the ratio $|p|_{\min } /|p|_{\text {adiab }}$ at free-molecular conditions, between the minimum pressure amplitude obtained in Fig. 7(b) and the counterpart pressure amplitude in response to adiabatic excitation. The thick, dashed thick, thin, and dashed thin curves present results for $\omega=3 \pi / 4,3 \pi / 2,3 \pi$, and $6 \pi$, respectively.

As discussed in Sec. IV A 2, the continuum-limit response is dominated by a single-frequency compression wave, for both animation and thermal excitations. It is therefore reasonable to expect that the conditions for optimal cancellation do not vary with the radial distance. In marked difference, the ballistic-flow expression for the acoustic pressure in Eq. (53) shows that it composes several decaying-wave contributions [see Eqs. (54) and (55)], each characterized by different phase velocity and decay rate. Consequently, the conditions for optimal sound cancellation vary with the distance from the boundary. It is only at very large distances from the source (outside the radial interval presented in Fig. 7) that the pressure is dominated by the most slowly decaying moment $K_{5}$, for which complete sound reduction is achieved at $\left|\tilde{Q}_{1} / \tilde{U}_{1}\right|^{\text {opt }}=1 / 8$. Yet, this result is of limited practical significance, since at such large distances from the source the acoustic signal is already vanishingly small. Inspecting Fig. 7(c), we find that the most efficient cancellation is achieved closest to the wall, where all wave components compounding the pressure are nearly in-phase. Moving away from the boundary to a distance $\approx 4-12$ acoustic wavelengths, the results indicate that the adiabatic-wall signal may be reduced by $\approx 50 \%$ (equivalent to $\mathrm{a} \approx 6 \mathrm{~dB}$ reduction in sound pressure level) via optimal choice of $\left|\tilde{Q}_{1} / \tilde{U}_{1}\right|$. This is, again, different from the continuum-limit results, according to which complete sound cancellation is achieved when applying the above $\gamma /[2(\gamma-1)]$ ratio. 


\section{CONCLUSION}

We studied the acoustic field of a circular cylinder immersed in a rarefied gas and subject to harmonic small-amplitude normal-to-wall displacement and heat-flux excitations. The problem was analyzed in the entire range of gas rarefaction rates and excitation frequencies, considering both single cylinder and coaxial cylinders setups. Numerical calculations were carried out using the direct simulation Monte Carlo method, where a noniterative algorithm to impose boundary heat-flux conditions was applied. Analytical solutions were obtained in the limits of ballisticand continuum-flow conditions, and validated via Monte Carlo simulations. Comparing with the reference continuum inviscid solution, the results obtained present the specific impacts of gas rarefaction and boundary curvature on the acoustic source efficiency. Inspecting the decay properties of the generated disturbance, the continuum-limit solution shows on an exponential decay of the signal with the distance from the source, reflecting the thermoviscous effect. This is accompanied by an inverse square root decay with the propagation distance, characteristic of the inviscid problem. Stronger attenuation was observed in the ballistic limit, where the signal vanishes at a distance of few acoustic wavelengths from the boundary and becomes confined to a narrower layer with increasing frequency. The combined impacts of mechanical and thermal excitations were studied to seek for optimal conditions to minimize the vibroacoustic signal. The effect of boundary curvature on the optimal conditions becomes significant in the ballistic-flow regime, where the optimal heatflux amplitude required for sound reduction decreases with the distance from the source, and essentially depends on the acoustic-wavelength-scaled distance only. Comparing between the DSMC and analytic predictions, the limits of validity of the free-molecular and continuum schemes were identified and rationalized, and the respective differences between the single- and double-cylinder setups were discussed.

By considering the acoustic radiation of a pulsating cylinder, the present work aims at analyzing the effect of continuum breakdown on the efficiency of a two-dimensional acoustic monopole in a relatively simple model setup. While the work focuses on the system response to harmonic excitation, it could be easily extended to study the acoustic field generated by arbitrary smallamplitude excitation of the boundary, treating the current analysis as a generalized Fourier component of the input signal. A desirable extension of our analysis, investigating the counterpart problem in other non-planar setups of interest, is currently underway.

\section{ACKNOWLEDGMENT}

This research was supported by the Israel Science Foundation (Grant No. 1084/16).

\section{APPENDIX: EVALUATION OF $\boldsymbol{G}_{\boldsymbol{n}}$ IN EQ. (54)}

To evaluate the $\xi_{2 \mathrm{D}}$-integral in $G_{n}$ in Eq. (54), we make use of the steepest-descent analysis carried out by Abramowitz [28]. The calculation, formally valid for $\omega(r-1) \gg 1$, yields

$$
\begin{aligned}
G_{n} \approx & \operatorname{Re}\left\{\frac{1}{\sqrt{3^{n+1} \pi}} \exp [i \omega t] \int_{-\varphi}^{\varphi}\left(z \cdot g_{1}^{2 / 3}\right)^{n / 2}\left[1+\frac{a_{1}}{\left(z \cdot g_{1}^{2 / 3}\right)}+\frac{a_{2}}{\left(z \cdot g_{1}^{2 / 3}\right)^{2}}\right]\right. \\
& \left.\times \exp \left[-\left(z \cdot g_{1}^{2 / 3}\right)\right] d \theta_{\xi}\right\},
\end{aligned}
$$

where

$$
\begin{aligned}
z & =3[\omega(r-1) / 2]^{2 / 3} \exp [i \pi / 3] \\
a_{1} & =\left(3 n^{2}+3 n-1\right) / 12, \quad a_{2}=\left(9 n^{4}+6 n^{3}-51 n^{2}-24 n+25\right) / 288
\end{aligned}
$$


The remaining $\theta_{\xi}$-integral is evaluated via the method of stationary phase. Expanding the integrand about the single stationary phase point $\theta_{\xi}=0$, we obtain

$$
G_{n} \approx \operatorname{Re}\left\{\sqrt{\frac{z^{n}}{3^{n+1} \pi}}\left(1+\frac{a_{1}}{z}+\frac{a_{2}}{z^{2}}\right) \exp [i \omega t-z] \int_{-\varphi}^{\varphi} \exp \left[\frac{1}{3} z r \theta_{\xi}^{2}\right] d \theta_{\xi}\right\} .
$$

The $\theta_{\xi}$-integration then yields Eq. (55).

[1] P. M. Morse, Vibration and Sound (McGraw-Hill, New York, 1948).

[2] K. Shariff and M. Wang, A numerical experiment to determine whether surface shear-stress fluctuations are a true sound source, Phys. Fluids 17, 107105 (2005).

[3] C. L. Morfey, S. V. Sorokin, and G. Gabard, The effects of viscosity on sound radiation near solid surfaces, J. Fluid Mech. 690, 441 (2012).

[4] L. Sirovich and J. K. Thurber, Propagation of forced sound waves in rarefied gasdynamics, J. Acoust. Soc. Am. 37, 329 (1965).

[5] S. K. Loyalka and T. C. Cheng, Sound-wave propagation in a rarefied gas, Phys. Fluids 22, 830 (1979).

[6] S. Stefanov, P. Gospodinov, and C. Cercignani, Monte Carlo simulation and Navier-Stokes finite difference calculation of unsteady-state rarefied gas flows, Phys. Fluids 10, 289 (1998).

[7] N. G. Hadjiconstantinou, Sound wave propagation in transition-regime micro- and nanochannels, Phys. Fluids 14, 802 (2002).

[8] D. Kalempa and F. Sharipov, Sound propagation through a rarefied gas confined between source and receptor at arbitrary Knudsen number and sound frequency, Phys. Fluids 21, 103601 (2009).

[9] A. Manela, G. A. Radtke, and L. Pogorelyuk, On the damping effect of gas rarefaction on propagation of acoustic waves in a microchannel, Phys. Fluids 26, 032001 (2014).

[10] Y. Sone, Effect of sudden change of wall temperature in rarefied gas, J. Phys. Soc. Jpn. 20, 222 (1965).

[11] D. C. Wadsworth, D. A. Erwin, and E. P. Muntz, Transient motion of a confined rarefied gas due to wall heating or cooling, J. Fluid Mech. 248, 219 (1993).

[12] A. Manela and N. G. Hadjiconstantinou, On the motion induced in a gas confined in a small-scale gap due to instantaneous boundary heating, J. Fluid Mech. 593, 453 (2007).

[13] A. Manela and N. G. Hadjiconstantinou, Gas-flow animation by unsteady heating in a microchannel, Phys. Fluids 22, 062001 (2010).

[14] A. Manela and L. Pogorelyuk, Cloaking via heating: approach to acoustic cloaking of an actuated boundary in a rarefied gas, Phys. Fluids 26, 062003 (2014).

[15] A. Manela and L. Pogorelyuk, Active noise control of a vibrating surface: Continuum and non-continuum investigations on vibroacoustic sound reduction by a secondary heat-flux source, J. Sound Vib. 358, 20 (2015).

[16] Y. W. Yap and E. Sader, Sphere oscillating in a rarefied gas, J. Fluid Mech. 794, 109 (2016).

[17] D. Kalempa and F. Sharipov, Numerical modeling of thermoacoustic waves in a rarefied gas confined between coaxial cylinders, Vacuum 109, 326 (2014).

[18] S. Ramanathan, D. L. Koch, and R. B. Bhiladvala, Noncontinuum drag force on a nanowire vibrating normal to a wall: Simulations and theory, Phys. Fluids 22, 103101 (2010).

[19] P. Gospodinov, V. Roussinov, and S. Stefanov, Nonisothermal oscillatory cylindrical couette gas flow in the slip regime: A computational study, Eur. J. Mech. B-Fluids 33, 14 (2012).

[20] J. Meng, Y. Zhang, and J. M. Reese, Numerical simulation of rarefied gas flows with specified heat flux boundary conditions, Commun. Comput. Phys. 17, 1185 (2015).

[21] A. Manela and L. Pogorelyuk, Instantaneous insulation in a micro-slab: A mechanism for flow generation in a rarefied gas, Phys. Rev. Fluids 1, 084102 (2016).

[22] G. Bird, Molecular Gas Dynamics and the Direct Simulation of Gas Flows (Clarendon, Oxford, 1994). 
[23] Q. Wang, X. Yan, and Q. He, Heat-flux-specified boundary treatment for gas flow and heat transfer in microchannel using direct simulation Monte Carlo method, Int. J. Numer. Methods Eng. 74, 1109 (2008).

[24] H. Akhlaghi and E. Roohi, A novel algorithm for implementing a specified wall heat flux in dsmc: Application to micro/nano flows and hypersonic flows, Comput. Fluids 127, 78 (2016).

[25] M. N. Kogan, Rarefied Gas Dynamics (Plenum, New York, 1969).

[26] Y. Sone, Molecular Gas Dynamics: Theory, Techniques, and Applications (Birkhäuser, Boston, 2007).

[27] J. W. S. Rayleigh, The Theory of Sound (Macmillan, London, 1877), Vol. 1.

[28] M. Abramowitz, Evaluation of the integral $\int_{0}^{\infty} e^{-u^{2}-x / u} d u$, J. Math. Phys. 32, 188 (1953).

[29] W. H. Lin and A. C. Raptis, Thermoviscous effects on acoustic scattering by thermoelastic solid cylinders and spheres, J. Acoust. Soc. Am. 74, 1542 (1983). 